Draft June 20, 2018

\title{
ULTRAVIOLET SPECTROSCOPY OF RAPIDLY ROTATING SOLAR-MASS STARS: EMISSION-LINE REDSHIFTS AS A TEST OF THE SOLAR-STELLAR CONNECTION
}

\author{
Jeffrey L. Linsky ${ }^{1}$ \\ JILA, University of Colorado and NIST, 440UCB Boulder, CO 80309-0440, USA \\ jlinsky@jilau1.colorado.edu \\ Rachel Bushinsky \\ APS, University of Colorado, 391UCB Boulder, CO 80309-0391, USA \\ Tom Ayres ${ }^{1}$ \\ CASA, University of Colorado, 593UCB Boulder, CO 80309-0593, USA \\ and \\ Kevin France \\ CASA, University of Colorado, 593UCB Boulder, CO 80309-0593, USA
}

\begin{abstract}
We compare high-resolution ultraviolet spectra of the Sun and thirteen solarmass main sequence stars with different rotational periods that serve as proxies for their different ages and magnetic field structures. In this the second paper in the series, we study the dependence of ultraviolet emission-line centroid velocities on stellar rotation period, as rotation rates decrease from that of the Pleiades star HII314 ( $P_{\text {rot }}=1.47$ days) to $\alpha$ Cen A ( $P_{\text {rot }}=28$ days). Our stellar sample of F9 V to G5 V stars consists of six stars observed with the Cosmic Origins
\end{abstract}

\footnotetext{
${ }^{1}$ Guest Observer, NASA/ESA Hubble Space Telescope and User of the Data Archive at the Space Telescope Science Institute. STScI is operated by the Association of Universities for Research in Astronomy, Inc., under NASA contract NAS 5-26555. These observations are associated with programs \#11532, \#11534, and \#11687.
} 
Spectrograph on HST and eight stars observed with the Space Telescope Imaging Spectrograph on HST. We find a systematic trend of increasing redshift with more rapid rotation (decreasing rotation period) that is similar to the increase in line red shift between quiet and plage regions on the Sun. The fastest-rotating solar-mass star in our study, HII314, shows significantly enhanced redshifts at all temperatures above $\log T=4.6$, including the corona, which is very different from the redshift pattern observed in the more slowly-rotating stars. This difference in the redshift pattern suggests that a qualitative change in the magnetic-heating process occurs near $P_{\text {rot }}=2$ days. We propose that HII314 is an example of a solar-mass star with a magnetic heating rate too large for the physical processes responsible for the redshift pattern to operate in the same way as for the more slowly rotating stars. HII314 may therefore lie above the high activity end of the set of solar-like phenomena that is often called the "solar-stellar connection".

Subject headings: stars: chromospheres — stars: individual (HII314, EK Dra, $\zeta$ Dor, $\pi^{1}$ UMa, $\chi^{1}$ Ori, HD 165185, HD 25825, HD 97334, $\kappa^{1}$ Cet, HD 73350, HD 59967, HD 209458, Sun, $\alpha$ Cen A) — stars:solar-type — Sun:chromosphere — Sun:UV radiation

\section{INTRODUCTION}

After their pre-main sequence evolution, solar-mass stars reach the zero-age main sequence as rapid rotators with strong magnetic fields. As they age on the main sequence, the torque of their magnetized winds slows their rotation rate (e.g., Matt \& Pudritz 2008), producing a wide range of changes in their magnetic structure and emission properties. Wilson (1963) and Kraft (1967), among others, showed that the strength of the chromospheric Ca II lines decreases with age and rotational velocity for solar-type stars. Other changes also occur in the X-ray and radio emission, starspot properties, and magnetic field structure. In this series of papers, we explore the evolution of the ultraviolet spectra of solar-mass stars as they slow down with age on the main sequence.

In a series of four papers, we measure and interpret the properties of ultraviolet emission lines and continua formed above the photospheres of a sample of solar-mass dwarf stars that differ primarily in the rotation rates that govern their magnetic activity. The emission lines are formed in the chromospheres and higher atmospheric layers that are heated by magnetic processes that weaken as slower rotation decreases the dynamo generation of magnetic fields in these convective stars. The properties of the emission lines provide qualitative measures of the magnetic heating rates. This stellar intercomparison, using rotation period as the 
independent variable, is now feasible because of the availability of high-resolution spectra of 13 solar-mass stars observed with the Cosmic Origins Spectrograph (COS) and Space Telescope Imaging Spectrograph (STIS) on the Hubble Space Telescope (HST). Our intercomparison of solar-mass stars follows on the "Sleuthing the Dynamo" study of young cluster stars (Ayres et al. 1996; Ayres 1999), which was based on lower-resolution UV spectra obtained with first generation instruments on HST. Although the age and rotation dependence of the ultraviolet and X-ray emission of solar-mass stars, often referred to as the "Sun in Time" project, is an active field of research (e.g., Ribas et al. 2005; Pizzolato et al. 2003; Simon et al. 1985), we provide a new and more detailed perspective using high-resolution spectra.

In the first paper of the series (Linsky et al. 2012), we measured the continuum flux between the emission lines in the 1150-1500 $\AA$ region for seven solar-mass stars and the Sun. We found that the continuum brightness temperatures $\left(T_{B}\right)$ increase systematically with increasing rotation rate: the $T_{B}$ of the slowest rotator $(\alpha$ Cen $\mathrm{A}$ ) is similar to that of solar regions with very weak magnetic fields, and the $T_{B}$ of the fastest rotators (HII314 and EK Dra) are similar to solar regions with strong magnetic fields (bright plages). Thus the $T_{B}$ range seen in solar-mass stars with rotation periods $\left(P_{\text {rot }}\right)$ between 1.47 and 28 days corresponds to the range in $T_{B}$ seen from lowest to highest magnetic-field regions on the Sun. This result provides a beautiful example of the solar-stellar connection.

In the next three papers in the series, we explore three other aspects of the solar-stellar connection. In the present paper, we measure the wavelength shifts of ultraviolet emission lines in a sample of 14 solar-mass stars including the Sun to study the dependence of redshift on rotation period. In the next two papers, we will study the line-flux saturation and linewidth dependencies on rotation period. A major question that we will address is whether these four indicators of stellar magnetic activity are consistent in that they show roughly the same dependence on stellar rotation velocity.

The decrease in ultraviolet emission-line, X-ray, and EUV fluxes as stars age on the main sequence has often been characterized by power laws. For example, Skumanich (1972) proposed that the chromospheric Ca II line flux and the rotation velocity both decay with time as $t^{-1 / 2}$. In their analysis of the early IUE observations, Ayres et al. (1981) showed that the fluxes of chromospheric and transition region lines and the X-ray fluxes of spectral type $\mathrm{F}-\mathrm{K}$ dwarf stars are correlated with different power law slopes, indicating that agedependent heating occurs in the various atmospheric layers but at different rates and perhaps by different processes. Subsequent studies of solar-type main sequence stars [e.g., Avres et al. (1996); Cardini \& Cassatella (2007)] have provided more accurate slopes to the power-law correlations by using more extensive and higher quality UV and X-ray data sets. In their 
comprehensive study of the $1-1700 \AA$ irradiance of 6 solar-mass stars and the Sun covering ages $(0.5-1.0) \times 10^{8} \mathrm{yr}($ EK Dra $)$ to $6.7 \times 10^{9} \mathrm{yr}(\beta \mathrm{Hyi})$, Ribas et al. (2005) obtained power law relations, $F=a t^{-b}$, between radiative flux in different wavelength bands and stellar age $(t)$. For the integrated flux of UV emission lines, the power-law index increases from about $b=0.70$ for low chromosphere lines (C I, H I, and O I) to about $b=1.0$ for hot transition region lines ( $\mathrm{Si} \mathrm{IV}, \mathrm{C} \mathrm{IV}$, and $\mathrm{O}$ VI). Thus the young rapidly-rotating Sun at age $10^{8} \mathrm{yr}$ likely had fluxes relative to the present Sun enhanced by a factor of 30 for chromospheric emission lines to 1000 times larger for X-rays.

There is empirical evidence that magnetic-field structures are qualitatively different in very active solar-mass stars compared to less active stars. While the Sun never shows sunspots at the pole or at very high latitudes, $\xi$ Boo A (G8 V), the solar-like star just to the right of the break in the mass flux-X-ray correlation (Wood et al. 2005), shows highlatitude spots (Toner \& Grav 1988) and Zeeman-Doppler images (ZDI) show an inclined dipole and a large-scale toroidal magnetic field (Petit et al. 2005) that is very different from the Sun. This pattern of a dipolar magnetic field with a torroidal component is detected in ZDIs of other rapidly rotating solar-mass stars (Donati \& Landstreet 2009). Simulations by Schrijver \& Title (2001) of the magnetic field in very active Sun-like stars that differ from the Sun only by the imposed rate of magnetic flux emerging into the photosphere from below show a major change from the solar magnetic-field pattern. When the imposed rate of magnetic-field emergence is 30 times that of the Sun, a strong magnetic-field polar cap forms surrounded by a torroidal ring of opposite polarity. The polar cap likely includes large starspot clusters. The magnetic flux on the surface of this very active Sun-like star is similar to that of a solar-mass stars with $P_{\text {rot }}=6$ days. The rotation period of $\xi$ Boo A is 6.2 days (Noyes et al. 1984).

The observations just summarized point to a qualitative difference between the magneticfield structure of rapidly rotating solar-mass stars compared to slowly rotating stars like the Sun; the magnetic field simulations confirm this difference. Given the qualitative difference in magnetic-field structure, one might expect that the kinematics of warm plasma in the atmosphere layers between the photosphere and corona would also be very different, because a major fraction of the energy from magnetic-field reconnection events is converted into heat that is then radiated from these atmosphere layers. Until recently, only a few F- and G-type stars were bright enough to be studied with high-resolution UV spectroscopy - $\alpha$ Cen A (Wood et al. 1997; Pagano et al. 2004), Procyon (Wood et al. 1997), $\zeta$ Dor (Redfield et al. 2001), and EK Dra (Ayres \& France 2010). These studies raised many of the questions that form the basis for the present systematic study of stars very close in spectral type and mass to the Sun. The very efficient COS instrument on HST has increased the number solar-mass stars available for this study, in particular, the more distant rapidly rotating stars like HII314 
located in the Pleiades cluster at 134 pc.

In Section 2 we describe the observations obtained with COS and STIS that form our data base. Section 3 describes our procedures for extracting emission-line redshifts, and Section 4 compares the stellar and solar redshifts. In Section 5, we summarize our results.

\section{DATA SET AND HST OBSERVATIONS}

We have searched the MAST1 archive and the StarCAT STIS spectral catalog Ayres 2010) for UV spectra (1150-1600 ^) of solar-mass stars, which for this study are defined as those stars with spectral types between F9 V and G5 V. These spectral types correspond to the mass range $0.92-1.1$ solar masses. We identify 13 stars observed with the COS G130M and STIS E140M and E140H gratings that, including the Sun, we call the high-resolution group (see Tables 1 and 2). One star, $\chi^{1}$ Ori, was observed by both COS and STIS. The spectral resolving powers $R=\lambda / \Delta \lambda=17,000$ (COS G130M), 45,000 (STIS E140M), and 114,000 (STIS E140H) allow us to analyze line-profile shapes and Doppler shifts. Tables 1 and 2 provide information on the observing programs, stellar ages, cluster memberships, rotation periods $\left(P_{\text {rot }}\right)$, rotation velocities $(v$ sini), and spectral types of stars in both groups.

Our collection of solar-mass stars includes the stars observed with IUE in the "Sun in Time" program of Ribas et al. (2005) and the young stars observed by Ayres et al. (1996) and Ayres (1999) with the GHRS G140L and FOS G130H gratings. We include only those stars with usable $\mathrm{S} / \mathrm{N}$ in the observed UV emission lines.

The Pleiades Cluster G1 V star HII314 was selected for COS observations because it has the highest UV line fluxes of a solar mass star in the Avres (1999) sample. We adopt the rotational period $P_{\text {rot }}=1.47$ days obtained by Rice \& Strassmeier $(2001)$ in their Doppler-imaging study of the star. EK Dra $\left[P_{\text {rot }}=2.6050 \pm 0.0003\right.$ days, Strassmeier \& Rice $(1998)]$ is a member of the Pleiades moving group. $\pi^{1} \mathrm{UMa}\left[P_{\mathrm{rot}}=4.89\right.$ days, Gaidos et al. (2000) $], \chi^{1}$ Ori $\left[P_{\text {rot }}=5.104\right.$ days, King \& Schuler $\left.(2005)\right]$, and HD $165185\left[P_{\text {rot }}=5.90\right.$ days, Saar \& Osten (1997)] are members of the Ursa Major moving group. HD 25825 is a member of the Hyades cluster, and $\zeta$ Dor $\left[v\right.$ sini $=14.8 \mathrm{~km} \mathrm{~s}^{-1}$ corresponding to $P_{\text {rot }} \leq 4.0$ days if $\left.R_{\star} / R_{\odot}=1.15\right)$ Schroeder et al. (2009)], $\kappa^{1}$ Cet $\left[P_{\text {rot }}=9.24\right.$ days, Barnes (2007) $]$, HD $97334\left[P_{\text {rot }}=7.60\right.$ days, Hempelmann et al. (1995)], HD 73350, HD 59967, HD 209458, and $\alpha$ Cen $\mathrm{A}\left[P_{\text {rot }}=28 \pm 3\right.$ days, Barnes 2007] are field stars. The rotational period of 11.4 days for HD 209458 was measured by Silva-Valio (2008) from occultations of starspots by

\footnotetext{
${ }^{1}$ http://archive.stsci.edu
} 
its transiting planet. We estimate that the rotational period for HD 25825 is $P_{\text {rot }} \approx 6.5$ days based on the measured periods of Hyades G0 V stars (Radick et al. 1987), and the mean period for stars with $\mathrm{B}-\mathrm{V}=0.60$ and Hyades age (Barnes 2010). Rotational periods for the other stars were obtained from Pizzolato et al. (2003).

The HST spectra were obtained from several programs. HII314 was observed by COS in Program 11532 (J. Green PI). EK Dra, $\pi^{1}$ UMa, $\chi^{1}$ Ori and HD 25825 were observed as part of the Fe XXI emission line SNAP program 11687 (T. Ayres PI). HD 209458 was observed in program 11534 (J. Green PI) by Linsky et al. (2010) to study its transiting planet. The STIS E140H spectrum of $\alpha$ Cen A is published by Pagano et al. (2004), and the STIS E140M spectra of $\chi^{1}$ Ori and $\kappa$ Cet were obtained from the HST archive. Table 3 is the observing $\log$ for these COS and STIS observations.

\subsection{COS Observations}

A description of the COS instrument and on-orbit performance can be found in Green et al. (2102) and Osterman et al. (2011). We observed HII314 (V1038 Tau) with the COS G130M grating on 2009 December 16 for a total of 3209 seconds. We requested observations for four orbits, but on the last orbit HST failed to reacquire the target on time, leading to a blank spectrum. We observed HII314 with three central wavelength settings $(\lambda 1300, \lambda 1309$, and $\lambda 1318)$ to minimize fixed-pattern noise in the detector. The resulting spectral range was $1134 \leq \lambda \leq 1459$, with a resolving power of $R \approx 17,000-18,000$. On 2010, October 11 we reobserved HII314 using the G160M grating.

We retrieved the calibrated COS data files from MAST 22 and cross-correlated the individual data files obtained for the different grating settings to determine a common wavelength scale using the COADD_x1d.pro software package written in IDL by Charles Danforth. We found it necessary to reprocess the far-UV observations with this custom version of CALCOS3 v2.11, because incomplete pulse-height screening produced residual spurious features in the coadded spectra. The COS G130M spectrum is presented in Figure 1 together with other COS and STIS spectra.

The SNAP program consisted of single orbit observations using only the COS G130 grating at the $\lambda 1291$ central wavelength setting that covered the wavelength region 1290-

\footnotetext{
${ }^{2}$ http://archive.stsci.edu

${ }^{3}$ We refer the reader to the cycle 18 COS Instrument Handbook for more details: http://www.stsci.edu/hst/cos/documents/handbooks/current/cos_cover.html
} 
$1430 \AA$. For most of the SNAP observations, the $1134-1274 \AA$ region of the detector was turned off to avoid overexposure in the Lyman- $\alpha$ line. Exposure times for the four stars in the SNAP program selected for this study were between 1160 and 1300 seconds. We reduced these data in the same way as described for HII314, except that there was only one wavelength setting, although FP-POS positions 3 and 4 were utilized.

We also include the combined out-of-transit observations of HD 209458 consisting of 20,694 seconds of exposure at both orbital quadratures and secondary eclipse of HD 209458b. These observations are described by France et al. (2010b) and Linsky et al. (2010).

\subsection{STIS Observations}

We include in this study STIS moderate resolution E140M spectra of the field stars $\zeta$ Dor, HD 97334, HD 73350, HD 59967, and $\kappa^{1}$ Cet and the Ursa Major moving group stars HD 165185 and $\chi^{1}$ Ori, which was also observed with COS. The STIS observations were obtained with program 8280 (PI T. Ayres) using the $0.2 \times 0.2$ aperture. The spectra cover the range $1140-1729 \AA$ with a spectral resolution of $\mathrm{R} \approx 40,000 . \kappa^{1}$ Cet was observed for 7805 seconds on September 19, 2000, and $\chi^{1}$ Ori was observed by STIS for 6770 seconds on October 3, 2000. We retrieved the STIS spectra from StarCAT4 provided by Thomas Ayres. These spectra have well-calibrated flux and wavelength scales and did not require further processing. Figure 1 shows spectra of ten solar-mass stars observed by COS and STIS. Included for comparison is the STIS E140H spectrum of the slowly rotating near solar twin $\alpha$ Cen A (Pagano et al. 2004). Although the spectra appear very similar, the flux scales increase with more rapid rotation. We will discuss this increase in emission and the resulting saturation of line fluxes in a subsequent paper using these observations.

$\alpha$ Cen A was recently reobserved by STIS using the E140M and E140H gratings. Results from this observing program led by T. Ayres will be presented elsewhere, but we include here measurements of the radial velocities of the Si IV and C IV lines.

\subsection{Comparison Solar Irradiance Measurements}

We compare the stellar observations of solar mass stars with solar irradiance measurements, which are flux values of the Sun viewed as a distant point source like our stellar flux measurements. We use the solar-irradiance reference spectra obtained with the Solar

\footnotetext{
${ }^{4}$ http://casa.colorado.edu/ ayres/StarCAT/
} 
Radiation and Climate Experiment (SORCE) on the Solar-Stellar Irradiance Comparison Experiment II (SOLSTICE II) (Woods et al. 2009; McClintock et al. 2005; Snow et al. 2005). These data cover the $1150-3200 \AA$ spectral range with $1.0 \AA$ resolution. We selected this data set because the absolute flux calibration is accurate to about $5 \%$ in the UV and is crosschecked against B and A-type stars. Scattered and stray light are removed from these data. We use here the March 25-29, 2008 data set that represents the Sun close to minimum with a Zurich-sunspot number of 2 and an average $10.7-\mathrm{cm}$ radio flux of $69 \times 10^{-22} \mathrm{Wm}^{-2} \mathrm{~Hz}^{-1}$.

Although the Sun is $310^{12}$ times brighter than, for example, $\chi^{1}$ Ori, the relative number of photons observeable by SORCE and COS is only $10^{4}$ considering the different aperture sizes $(0.1 \times 0.1 \mathrm{~nm}$ for SORCE vs $2.4 \mathrm{~m}$ diameter for HST), observing times (180 seconds for SORCE vs 1300 seconds for COS), and resolution elements (0.1 nm for SORCE and $0.0076 \mathrm{~nm}$ for COS). If the two instruments have comparable efficiences, then the $\mathrm{S} / \mathrm{N}$ of the SORCE FUV spectra should be 30 times that of the COS FUV spectra binned to the SORCE resolution.

\subsection{Extraction of the Emission-Line Profile Parameters}

We extracted the intrinsic emission-line profile parameters (emission-line flux, FWHM, and central wavelength) from the COS and STIS spectra using the COSFIT-freedom.pro program written by Kevin France for the COS Science Team. We used this extraction procedure, as described by France et al. (2010a), to fit either single and double Gaussians to the line profiles. Both Gaussians were fitted empirically and convolved with the point spread function (PSF). We imposed no constraints on the central velocities of the Gaussians because previous solar and stellar studies show that both Gaussians are typically Doppler shifted from the photospheric radial velocity. We then converted the measured flux $f$ (units: ergs $\left.\mathrm{cm}^{-2} \mathrm{~s}^{-1}\right)$ to surface flux $F$ by $F=f(d / R)^{2}$. Values for the radius $(R)$, distance $(d)$, and stellar rotation period $\left(P_{\text {rot }}\right)$ are given in Tables 1 and 2 .

Figure 2 shows examples of the line-fitting procedure for several of the emission lines of HII314, $\kappa^{1}$ Cet and HD 209458. The dotted (red) lines are the intrinsic narrow and broad Gaussians, and the solid (blue) line is the convolution of the two Gaussians with the COS line-spread function that best fits the observed line profile. Although this procedure assumes that the intrinsic line profiles are single or double Gaussians in shape, the fits to the observed line profiles are generally very good even in the line wings. Note that the derived central wavelengths of the two Gaussians are not the same in these examples. Tables A1-A13 (available in the on line journal) list the laboratory wavelengths (Morton 1991;

Pagano et al. 2004) and derived-intrinsic line parameters for all measured emission lines 
from the stars. The COS and STIS data for $\chi^{1}$ Ori are listed in separate tables. Also listed are the heliocentric velocity shifts of the intrinsic Gaussian centroids compared to the laboratory wavelengths.

Since the STIS instrument was designed and calibrated to have an accurate velocity scale, the STIS velocity measurements are more accurate than for COS, which has a large aperture with no slit. For the STIS E140M grating data, the scatter in measured-velocity centroids for the photospheric lines of $\kappa^{1}$ Cet relative to the linear least-squares fit is about $1.5 \mathrm{~km} \mathrm{~s}^{-1}$, and the velocity slope between 1300 and $1400 \AA$ is less than $0.7 \mathrm{~km} \mathrm{~s}^{-1}$ and likely zero. The photospheric radial velocity of $\kappa^{1}$ Cet is listed in SIMBAD as $19.9 \pm 0.9 \mathrm{~km} \mathrm{~s}^{-1}$, as compared to the least-squares fit to the photospheric lines near $1350 \AA$ of $17.2 \mathrm{~km} \mathrm{~s}^{-1}$. The photospheric radial velocity for $\chi^{1}$ Ori measured from the neutral lines in the STIS data near $1350 \AA$ is $-15.77 \pm 0.17 \mathrm{~km} \mathrm{~s}^{-1}$ (see Table 4) as compared to the radial velocity listed in SIMBAD of $-13.5 \pm 0.9 \mathrm{~km} \mathrm{~s}^{-1}$. We adopt the $\mathrm{Cl} \mathrm{I} \lambda 1351.657$ line as our photosphericvelocity fiducial mark because it is a bright emission line well separated from possible blends, it is close in wavelength to the Si IV lines, and its measured radial velocity in the STIS $\chi^{1}$ Ori data (see Table 4) is $-15.51 \pm 0.35 \mathrm{~km} \mathrm{~s}^{-1}$, close to the mean velocity of the other photospheric lines.

Since the COS instrument was designed for maximum throughput at moderate spectral resolution but not with a precise wavelength scale, there could be wavelength-dependent velocity shifts in addition to velocity measurement errors for weak emission lines resulting from the modest spectral resolution. By comparison, STIS was designed to have an accurate velocity scale that was calibrated in the initial STIS data pipeline and then further refined by the StarCAT project (Ayres 2010).

To assess the wavelength dependence of the COS velocity scale in the important 1300$1400 \AA$ A region, which contains emission lines of Cl I, O I, C II, Si IV, Fe XII, and Fe XXI, we plot in Figure 4 the velocity difference between the COS and STIS measurements of the nine brightest emission lines that have COS velocity-measurement errors (random not systematic) of less than $2.0 \mathrm{~km} \mathrm{~s}^{-1}$. We have not included blended lines, the C II $\lambda 1334$ line that has intersellar absorption, or the O I $\lambda 1302,1304$, and 1306 lines that have strong geocoronal emission and interstellar absorption features. Figure 4 shows a $\sim 10 \mathrm{~km} \mathrm{~s}^{-1}$ linear increase in the COS velocity scale between 1300 and $1400 \AA$. The flux-weighted linear fit to these data is vel(fit) $=-148.392+0.113988 \lambda$. To correct for this slope in the COS velocity scale, we subtract $5.45 \mathrm{~km} \mathrm{~s}^{-1}$ from the COS v(Si IV) $-\mathrm{v}(\mathrm{Cl} \mathrm{I})$ measurements to force consistency between the COS and STIS velocities for $\chi^{1}$ Ori, the one star in common to both data sets. We assume that this calibration also applies to the other COS spectra, which are mostly SNAP observations observed with the same instrumental settings. We 
believe that this correction of the COS velocity scale in this wavelength interval should be the same for all of the COS observations because the geometry of the detector should not change as the high voltage was the same for all observations, and thermal drifts should be largely compensated by wavelength calibration lamp observations.

\subsection{Coronal Emission Lines}

In their search for forbidden coronal emission lines in STIS spectra of late-type stars and binary systems, Ayres et al. (2003) detected the Fe XXI $\lambda 1354.10$ and/or Fe XII $\lambda 1242.00$ lines in 19 stars, but they detected no other coronal emission lines. The Fe XXI and Fe XII lines were both detected in the active solar-mass stars $\chi^{1}$ Ori and $\xi$ Boo A and in several dMe stars. We therefore searched the COS spectra for coronal emission lines. We detected the Fe XXI line in HII314, EK Dra, $\pi^{1}$ UMa, and $\chi^{1}$ Ori, and the Fe XII $\lambda 1242.00$ line in $\zeta$ Dor, $\pi^{1}$ UMa, $\chi^{1}$ Ori, and HD209458. We also detected the Fe XII $\lambda 1349.36$ line in $\pi^{1}$ UMa and $\chi^{1}$ Ori. As noted by Ayres et al. (2003), there is a weak C I line at $1354.288 \AA(+41.6$ $\mathrm{km} \mathrm{s}^{-1}$ relative to the Fe XXI line) that must be unblended to obtain the most accurate velocity and FWMH of the Fe XXI line. We have made two-Gaussian fits to the blended Fe XXI and C I lines for EK Dra, $\pi^{1} \mathrm{UMa}$, and $\chi^{1}$ Ori. With increasing rotation rate, the Fe XXI line becomes stronger relative to the C I line until for HII314 the C I line is no longer measurable. For this star we fit the Fe XXI line with a single Gaussian. Figure 3 shows the observed and fitted profiles of the Fe XXI and Fe XII lines. We also measure $3 \sigma$ flux upper limits for the nondetected Fe XXI and Fe XII lines.

\section{EMISSION LINE REDSHIFTS}

Achour et al. (1995) and Peter \& Judge (1999) have summarized the observations of solar emission lines by many spacecraft that show line-centroid redshifts of UV emission lines relative to photospheric lines. The disk-center redshifts of emission lines formed in the solar chromosphere and transition region increase with line formation temperature up to $\log T \approx 5.3$ and then decrease rapidly with rising temperature. The ions with the largest redshifts are N V, O IV and O V formed at $\log T=5.2-5.4$. Achour et al. (1995) showed that in solar active regions, the Si IV and C IV lines show redshifts that are about $7 \mathrm{~km} \mathrm{~s}^{-1}$ larger than in quiet regions, indicating that the stronger magnetic fields in active regions are somehow enhancing the physical processes that produce the redshifts. There are a few measurements of stellar emission-line redshifts (e.g., Wood et al. 1997; Pagano et al. 2004; Ayres 2010), but until now there has been no systematic study of the redshift phenomenon 
in solar-mass stars.

In Table 5 we list the measured redshifts of the Si IV and C IV doublets for the 13 solarmass stars for which we have COS and STIS data. The Si IV velocities are flux-weighted averages of the central velocities of single-Gaussian fits to the Si IV $\lambda 1393$ and $\lambda 1402$ lines, and the $\mathrm{C}$ IV velocities are corresponding flux-weighted averages for the C IV $\lambda 1548$ and 1550 lines. These velocities are measured relative to the $\mathrm{Cl}$ I $\lambda 1351$ line, which we treat as representative of the photospheric velocity (see Section 2.4) to correct for errors in centering the star in the COS entrance aperture. Redshifts of the Si IV and C IV lines of $\alpha$ Cen A were measured differently. For each doublet, double-Gaussian profiles were constructed to best fit both lines together. The velocities listed in Table 5 for this star are flux-weighted averages of the broad and narrow Gaussians so as to be comparable with the other data in the table that are single-Gaussian fits.

Velocity errors are difficult to quantify as they consist of both measurement errors resulting from limited $\mathrm{S} / \mathrm{N}$, line asymmetries, and systematic errors that result from the imperfect instrument velocity scale and errors in the laboratory wavelengths. A rough estimate of the velocity errors is the dispersion in the measured centroid velocities of the $\lambda 1393$ and 1402 lines. The mean of this dispersion is $2.2 \mathrm{~km} \mathrm{~s}^{-1}$ for the COS data and $1.4 \mathrm{~km} \mathrm{~s}^{-1}$ for the STIS data. The standard deviation of the differences between the v(COS) - v(STIS) data and the weighted least-squares fit for the nine lines in Figure 4 is $1.87 \mathrm{~km} \mathrm{~s}^{-1}$. This standard deviation provides a rough measure of the velocity errors for the high and low $\mathrm{S} / \mathrm{N}$ lines in the COS data.

\section{DISCUSSION}

\subsection{IV and Si IV Redshifts vs. Stellar Rotational Periods}

Figure 5 shows a systematic decrease in the C IV and Si IV line redshifts measured relative to the $\mathrm{Cl}$ I reference line with increasing rotational period. This redshift-rotation correlation is indicated by linear least-squares fits to the STIS Si IV and C IV data separately and to the fit to the STIS and COS Si IV data taken together. All three fits show systematic decreases in redshift with rotational period, although the COS data show larger scatter about the fits. The mean errors for each data set are $2.3 \mathrm{~km} \mathrm{~s}^{-1}$ for the COS Si IV redshifts, 1.5 $\mathrm{km} \mathrm{s}^{-1}$ for the STIS Si IV redshifts, and $1.3 \mathrm{~km} \mathrm{~s}^{-1}$ for the STIS C IV redshifts.

Most measurements of solar UV emission lines are based on single-Gaussian fits to emission lines observed near disk center. We include in Table 5 the C IV and Si IV redshift measurements of Achour et al. (1995) obtained with the High Resolution Telescope and 
Spectrometer (HRTS) instrument. These are measurements of a quiet and an active region near disk center. We find that the Si IV redshift of the disk center quiet Sun $\left(+5.4 \mathrm{~km} \mathrm{~s}^{-1}\right)$ is similar to that of $\alpha$ Cen $\mathrm{A}\left(+4.7 \mathrm{~km} \mathrm{~s}^{-1}\right)$ and the more slowly rotating stars (HD 209458 and HD 73350), while the Si IV redshift of the disk center active Sun $\left(+11.0 \mathrm{~km} \mathrm{~s}^{-1}\right)$ is similar to that of EK Dra and rapidly rotating stars. C IV redshifts of quiet and active regions near disk center for the Sun $\left(+6.2\right.$ and $\left.+13.0 \mathrm{~km} \mathrm{~s}^{-1}\right)$ are also similar to the C IV redshifts of the slowly rotating and rapidly rotating solar-mass stars.

Since the stellar data are averages over the stellar disks including limb darkening or brightening and viewing bulk motions in the stellar atmosphere for all lines of sight, we need to estimate what the redshifts would be for the Sun viewed as a star. We refer to solar disk averages as "Sunstar" data. Peter (2006) presented full-disk line profiles of the C IV $\lambda 1548$ line obtained by summing raster scans of the whole solar disk obtained with the SUMER instrument on SOHO. The redshift of a single-Gaussian fit to the full disk C IV $\lambda 1548$ line is $+1.9 \mathrm{~km} \mathrm{~s}^{-1}$, whereas the corresponding fit to the disk-center line in the same data set is $+4.6 \mathrm{~km} \mathrm{~s}^{-1}$. He argued that the factor of 2.4 decrease in the redshift between solar disk center and Sunstar is due largely to viewing the mainly radial solar internal motions tangentially at the limb. Since there are no Sunstar data for the Si IV lines or for active regions covering the whole Sun, we assume that this factor of 2.4 also applies to the C IV and Si IV lines in the quiet and active Sun. We list in Table 5 and plot in Figure 5 Sunstar active and quiet redshifts estimated by dividing the disk-center redshifts of Achour et al. (1995) by this factor of 2.4 .

Figure 5 shows that the linear fit to the stellar C IV data for the Sun's rotation period predicts a redshift of $+4.0 \mathrm{~km} \mathrm{~s}^{-1}$, which lies somewhat above, but within measurement errors, of the Peter (2006) C IV Sunstar measurement $\left(+1.9 \mathrm{~km} \mathrm{~s}^{-1}\right)$ and consistent with the estimated redshifts for the C IV lines $\left(+2.6 \mathrm{~km} \mathrm{~s}^{-1}\right)$ and Si IV lines $\left(+2.3 \mathrm{~km} \mathrm{~s}^{-1}\right)$ based on the disk-center redshifts (see Table 5) divided by 2.4. The Sunstar C IV redshift velocity also lies below the observed $+4.6 \mathrm{~km} \mathrm{~s}^{-1}$ redshift for $\alpha$ Cen A and the $4.77 \mathrm{~km} \mathrm{~s}^{-1}$ redshift of the 12.3 day period star HD 73350, although the difference is likely within measurement errors. Peter (2006) suggested that larger redshifts seen in $\alpha$ Cen A could result from the $25 \%$ lower surface gravity of this star compared to the Sun. The estimated redshift for the active region of Sunstar corresponds to solar-mass stars with $P_{\text {rot }} \approx 10$ days.

Which comparison of solar to stellar redshifts is more realistic? The answer depends upon details of how the redshifts, which result from the heating and mass motions (driven by braiding of magnetic flux) in the MHD model of Peter et al. (2006), scale with increasing magnetic flux in the more rapidly rotating stars, and whether the the limb darkening/brightening behavior remains the same as solar for the more rapidly rotating stars that 
presumably are more covered by active regions. The answers to these questions require detailed models for the more rapidly rotating solar-mass stars, but we can now say that the magnitudes of the redshifts seen in solar-mass stars with rotation periods between 1.47 and 28 days are already seen on the Sun.

\subsection{Redshift Pattern vs. Stellar Rotational Period}

We plot in Figure 6 emission-line redshifts as a function of line-formation temperature (Pagano et al. 2004). The STIS velocities have no additional corrections, but the COS velocities are corrected for the linear slope of the COS velocity scale as described in Section 2.4. There is uncertainty in the laboratory wavelengths of the N V lines (Achour et al. 1995). The redshifts assuming the laboratory wavelengths of Hallin (1966) are systematically low as compared to emission lines formed at similar temperatures, but are systematically high using the Edlén (1934) wavelengths. We have therefore adopted the mean of the two measurements, which raises the $\mathrm{N}$ V redshifts systematically by $2.74 \mathrm{~km} \mathrm{~s}^{-1}$.

Figure 6 shows the redshift vs. temperature pattern relative to the photosphere represented by the $\mathrm{Cl}$ I $\lambda 1351$ line for the two fastest-rotating stars observed with COS, four more slowly-rotating stars observed with STIS and COS, $\alpha$ Cen A, and, for comparison, the solar disk-center data. All of the stars show a similar pattern of small redshifts in the chromosphere (O I), peak redshifts at line-formation temperatures somewhat above $10^{5} \mathrm{~K}$ (e.g., C IV and N V) and, except for HII314, no significant redshifts in the Fe XII and Fe XXI coronal lines. Typical measurement errors relative to the $\mathrm{Cl} \mathrm{I}$ line are $0.3-0.8 \mathrm{~km} \mathrm{~s}^{-1}$ for the bright lines (e.g., O I, C II, Si III, Si IV, and C IV) and 1.5-3.0 $\mathrm{km} \mathrm{s}^{-1}$ for the faint lines (e.g., O IV and O V) for the stars observed with STIS and EK Dra and $\pi^{1}$ UMa observed with COS. The faintest star observed with COS, HII314, shows larger measurement errors of 1.3-1.7 $\mathrm{km} \mathrm{s}^{-1}$ but $3.2 \mathrm{~km} \mathrm{~s}^{-1}$ for the faint $\mathrm{O} \mathrm{V} \lambda 1371$ line. Measurement errors for the faint Fe XII $\lambda 1242$ line are $2.5-3.7 \mathrm{~km} \mathrm{~s}^{-1}$. For the Fe XXI line the errors are about $2.5 \mathrm{~km} \mathrm{~s}^{-1}$ except for HII314 where the error is about $5 \mathrm{~km} \mathrm{~s}^{-1}$. Thus, except for HII314, the redshifts of the coronal lines are consistent with the radial velocity of the stellar photosphere.

A remarkable result shown in Figure 6 is that all of the solar-mass stars in our sample, except for HII314, show very similar redshift patterns in both magnitude and dependence on line formation temperature despite the wide range of $P_{\text {rot }}$ between 2.61 to 28 days and the resulting very different magnetic heating rates. For these stars the peak redshift is between 6.0 and $10.6 \mathrm{~km} \mathrm{~s}^{-1}$ occuring in the temperature range $\log T=4.8$ (Si III) to 5.25 (N V). EK Dra and $\zeta$ Dor have peak redshifts slightly above those of the more slowly-rotating stars. The coronal line redshifts for these stars are all consistent with $0 \mathrm{~km} \mathrm{~s}^{-1}$. 
The peak redshifts for HII314 are much larger $\left(14.2-16.26 \mathrm{~km} \mathrm{~s}^{-1}\right)$ than for the other stars and occur over the much larger temperature range $\log T=4.62$ (C II) to 6.95 (Fe XXI). This large qualitative difference between HII314 and the other stars is likely a consequence of the faster rotation of HII314. These results for HII314 suggest that for solar-mass stars rotating faster than about a two-day period, a qualitative break occurs from the usual solar-stellar connection in which increased magnetic heating increases the emission from the chromosphere and corona but not the kinematics or physical processes responsible for the redshift pattern. The different redshift pattern of HII314 suggests the appearance of different physical processes not seen in the slower-rotating solar-mass stars and thus the high activity end of the "solar-stellar connection".

We compare the stellar redshift results with the solar disk-center redshifts obtained by Peter \& Judge (1999) using the SUMER instrument on the SOHO spacecraft. Since these are disk-center data rather than Sunstar data, the amplitudes of the redshifts are likely smaller when averaged over the solar disk. If the C IV $\lambda 1548$ line is a reliable guide, the Sunstar redshifts may be roughly a factor of 2.4 smaller than the disk-center redshifts. The disk-center pattern of redshift vs. line-formation temperature is similar to that seen in all of the stars except HII314. The detection of solar blueshifts in several coronal lines identifies the switch from redshift to blueshift near $\log T \approx 5.7$ as the Ne VIII $(\log T=5.81)$ and

$\mathrm{Mg} \mathrm{X}(\log T=6.04)$ lines show small blueshifts, $-2.7 \pm 1.1$ and $-4.5 \pm 1.3$, respectively (Peter \& Judge 1999). The measurement errors in the stellar Fe XII and Fe XXI redshifts are too large to say whether the stellar lines have zero or slightly negative velocities.

\subsection{Redshifts of the Broad and Narrow Components}

Wood et al. (1997) analyzed high-resolution GHRS spectra of the Si IV and C IV lines of 12 stars including dwarfs, giants, spectroscopic binaries, and the Sun with spectral types F5 to M0. They found that (i) the observed line profiles could not be fit well by a single Doppler component, but could be fit better with both a narrow and a broad component, (ii) the fraction of the total line flux in the broad component increases with stellar activity, as measured by the X-ray surface flux, (iii) the central velocity of the narrow component is redshifted relative to the photospheric radial velocity, and (iv) the central velocity of the broad component can be either blueshifted or redshifted relative to the narrow component for different stars. They were concerned, however, that these results and the possible explanations that they explored could be compromised by the range of stellar properties (mass, spectral type, gravity, binarity, rotational velocity) in their sample of stars. They stated that "ideally one should observe stars with the same spectral type, but different activity levels". 
Our sample of stars meets this criterion.

We plot in Figure 7 the velocity differences between the broad and narrow component centroids of the two Gaussian fits to the C IV $\lambda 1548$ and Si IV $\lambda 1393$ lines for the stars observed by STIS. We do not include velocity differences measured with COS, because the STIS data have higher quality for this critical measurement as a result of higher $\mathrm{S} / \mathrm{N}$ due to their long exposure times and more precise knowledge of the line-spread function. The mean velocity difference for the C IV line observed in stars with $P_{\text {rot }}$ between 4 and 8.77 days is $5.57 \mathrm{~km} \mathrm{~s}^{-1}$, with no discernable variation with $P_{\text {rot }}$. Remarkably, this mean velocity is essentially the same as the Sunstar value of $5.1 \mathrm{~km} \mathrm{~s}^{-1}$ measured by Peter (2006), despite the Sun's much slower rotation. The corresponding mean value for the Si IV lines is 1.83 $\mathrm{km} \mathrm{s}^{-1}$ with somewhat larger scatter.

Inclusion of the new STIS observations of $\alpha$ Cen A changes our interpretation of the velocity difference data. The new data are consistent with the Pagano et al. (2004) measurements but more precise: $v_{b}-v_{n}=-1.1 \pm 0.3 \mathrm{~km} \mathrm{~s}^{-1}$ for the Si IV doublet and $-1.9 \pm 0.3$ for the C IV doublet. The least squares linear fits to the velocity difference data including $\alpha$ Cen A (solid lines in Figure 7) show strong increases to shorter rotation periods. We consider these fits more realistic than the mean values for stars in the limited rotation period range 4.0 to 8.77 days because they include the excellent $\alpha$ Cen A data. However, the Sunstar C IV velocity difference $\left(+5.1 \mathrm{~km} \mathrm{~s}^{-1}\right)$ is inconsistent with the $\alpha$ Cen A data and the fits to all of the data. Our conclusion that the velocity differences increase with decreasing rotational period should be tested by new STIS observations of other slowly-rotating solar-mass dwarf stars and new measurements of the C IV and Si IV velocity difference for the Sun viewed as a star (Sunstar). It is also possible, as suggested by Peter (2006), that the negative velocity differences observed in $\alpha$ Cen A might result from the lower gravity of $\alpha$ Cen A compared to the Sun and possible differences in its convection pattern.

The mean fraction of the total flux in the broad component of the $\mathrm{C}$ IV line for the STIS stars is $0.52 \pm 0.04$ with a range of 0.37 to 0.65 . The Sunstar value is 0.48 , consistent with the mean value.

\subsection{Physical Models for Redshifts}

The measured redshifts of UV emission lines of solar-mass stars are consistent with the pattern shown in solar disk-center data (Peter \& Judge 1999) with peak redshifts shown in lines formed near $\log T=5.3$ and decreasing redshifts for lines formed at higher temperatures. The Doppler shifts for stellar coronal lines are consistent with either no shifts or small 
blueshifts as are seen in the solar data. This pattern is seen in all of the stars except for the most rapidly-rotating star HII314. On purely observational grounds, Peter \& Judge (1999) argued that the best explanation for the solar Doppler shift pattern involves heating below the corona that produces waves or flows that propagate downwards into the transition region and upwards into the corona. Since gas confined in magnetic loops is a major source of solar UV and X-ray emission, the heating in loops likely occurs in one leg causing coronal gas to propagate upward along the loop, subsequently cool, and rain down as redshifted transition region plasma along the other leg of the loop. This type of explanation is now generally accepted for the solar data, and we propose that it is also valid for solar-mass stars.

A number of authors have proposed theoretical models to explain the observed solar Doppler shift pattern. We cite here two recent, but very different, models. Zacharias et al. (2011) developed a three-dimensional MHD model of the solar upper chromosphere and corona in which relatively cool chromospheric gas is pushed upwards and slowly heated by energy deposited at magnetic loop footpoints. This blueshifted radiation from the upflowing gas is relatively small compared to the redshifted radiation from the heated gas as it descends from the corona through the transition region with larger emission measure. This model, which is conceptually similar to the spicular model of Athay \& Holzer (1982), fits the average Doppler shifts of emission lines formed in the temperature range $\log T=4.5-5.5$, but does not explain blueshifts of coronal emission lines.

The three-dimensional MHD solar models computed by Hansteen et al. (2010) differ from the Zacharias et al. (2011) model in that the heating occurs primarily in the transition region by rapid, episodic events. The heating-induced overpressure produces downflows of the underlying plasma and upflows in the overlying coronal plasma. The effect is to produce redshifts of transition region lines like C IV and no or small blueshifts of coronal lines like Fe XII and Fe XV, consistent with solar-average line shifts and the stellar redshift data. An interesting result appears in their model for weak photospheric magnetic fields and minimal heating. They find no redshifts in this model and conclude that "transition region redshifts are a direct consequence to vigorous heating to coronal temperatures at low heights, with redshifts disappearing as the amplitude of the heating decreases." Since the UV emission-line fluxes (see Fig. 1), and thus heating, increase with rapid rotation, the trend of increasing Si IV and C IV redshifts with rapid rotation is in qualitative agreement with the Hansteen et al. (2010) models. We note that our estimate of the C IV redshift for a solar-mass star completely covered with solar network elements with their enhanced heating is similar to the observed redshift of EK Dra ( $P_{\text {rot }}=2.6050$ days), a solar-mass star with nearly 100 times the flux (and thus heating) in the Si IV lines. While additional calculations are needed to tie down the detailed connection between heating, transitionregion line emission, and redshifts, the Hansteen et al. (2010) calculations indicate that the 
heating mechanism in their models could explain both phenomena.

Wood et al. (1997) suggested that the broad component of emission lines formed in the chromosphere and transition region is produced by large motions associated with explosive events that may result from nanoflare heating. Peter (2001) proposed a very different model in which the broad components are produced by upward-propagating magneto-acoustic waves in coronal funnels. In the three-dimensional MHD models discussed by Hansteen et al. (2010), transient heating in the transition region occurs largely in spatially restricted regions where the added pressure produced by rapid heating generates high-speed mass motions. The sum of such events over the stellar surface could explain the observed broad components, in which case the fractional flux in the broad components is a measure of the transient-heating process.

\section{CONCLUSIONS}

In this systematic study of ultraviolet emission lines formed in the outer atmospheres of solar-mass dwarf stars, we investigate how the parameters of these emission lines depend upon a stellar rotation period between 1.47 to 28.0 days for stars that otherwize have similar parameters (e.g., mass, $T_{\text {eff }}, g$, chemical composition). This study is based on high-resolution ultraviolet spectra of six stars observed with the COS instrument on HST, eight stars observed with the STIS instrument on HST, and the full-disk solar C IV $\lambda 1548$ line profile. In this second paper of a series, we find the following results concerning emission-line redshifts:

(1) We find that increasing redshifts are correlated with more rapid rotation (decreasing $\left.P_{\text {rot }}\right)$ for the Si IV and C IV lines. The quiet Sun disk center Si IV and C IV redshifts lie slightly above, but are within measurements errors of, this trend, and the active Sun disk center redshifts are similar to those seen in EK Dra and other rapidly rotating stars. The observed solar full disk (Sunstar) redshift for the C IV $\lambda 1548$ line lies slightly below, but also within measurement errors of, least-squares fits to the other stars. New theoretical studies of how redshifts depend on the magnitude and direction of gas flows produced by magnetic heating are needed to determine how best to estimate solar full disk redshifts from disk center observations.

(2) The redshift pattern with line-formation temperature for EK Dra $\left(P_{\text {rot }}=2.61\right.$ days $)$ is similar to that of the more slowly rotating stars (including the Sun) with maximum redshift near $T=10^{5} \mathrm{~K}$ and no redshift of the coronal Fe XII and Fe XXI lines. This similarity indicates that the coronal plasma observed in the Fe XII and Fe XXI lines is not participating in a stellar wind but is instead constrained by magnetic fields to corotate with the stellar 
photosphere. Unlike the more slowly rotating stars, the fastest-rotating solar-mass star in our study, HII314, shows significantly enhanced redshifts at all temperatures above $\log T=4.6$, including the corona. This difference in the redshift pattern between HII314 and EK Dra suggests that a qualitative change in the magnetic-heating process occurs near $P_{\text {rot }}=2$ days. We propose that HII314 is an example of a solar-mass star that is rotating too fast with a magnetic heating rate that is too large for the physical processes that control the redshift pattern to operate in the same way as for the more slowly rotating stars. HII314 appears to lie above the high activity end of the set of solar-like phenomena that is typically called the "solar-stellar connection".

(3) The stronger emission lines in these stars are usually better fit by two Gaussians (one broad and the other narrow) rather than by a single Gaussian. We find that the mean velocity difference between the centroids of the broad and narrow components of the $\mathrm{C} \mathrm{IV}$ $\lambda 1548$ lines observed in stars with $P_{\text {rot }}$ between 4 and 8.77 days is $5.57 \mathrm{~km} \mathrm{~s}^{-1}$, with no discernable variation with $P_{\text {rot }}$. Remarkably, this mean velocity difference is essentially the same as the Sunstar value of $5.1 \mathrm{~km} \mathrm{~s}^{-1}$ measured by Peter (2006), despite the Sun's much slower rotation rate. The corresponding mean velocity difference for the Si IV lines is 1.83 $\mathrm{km} \mathrm{s}^{-1}$ with somewhat larger scatter.

The inclusion of new STIS spectra of $\alpha$ Cen A identifies a trend of increasing velocity differences with more rapid rotation. This result should be tested by new STIS observations of slowly rotating solar-mass stars and new measurements of the velocity differences for the Sun viewed as a star (Sunstar). The mean fraction of the total flux in the broad component of the C IV line is $0.52 \pm 0.04$ with a range of 0.37 to 0.65 . The Sunstar value is 0.48 , consistent with the mean value. The broad components could be the sum of many transient-heating events in which the added pressure produced by rapid heating generates high-speed mass motions (Hansteen et al. 2010). If this model is correct, then the fractional flux in the broad components is a measure of the transient heating process. We will return to this question in a later paper in this series in which we discuss the observed stellar line widths using the data in Tables A1-A13.

(4) Redshifts result from flows produced by magnetic heating. The observed pattern of small redshifts in lines formed in the chromosphere, maximum redshifts for lines formed in the temperature range $\log T=4.8-5.25$, and no redshifts or small blueshifts of coronal lines is consistent with the predictions of the three-dimensional MHD solar model of Hansteen et al. (2010). We suggest that the Hansteen et al. (2010) model be run at very large heating rates to determine whether the model can reproduce the different redshift pattern seen in HII314. This would be a good test of the applicability of the model for very high magnetic heating rates. 
This work is supported by NASA through grants NNX08AC146, NAS5-98043, and HSTGO-11687.01-A to the University of Colorado at Boulder. We thank Tom Woods and Martin Snow for providing the SORCE data and Steven Osterman for information on the calibration of COS.

Facilities: HST (COS), HST (STIS), SUMER/SOHO, SORCE/SOLSTICE II, SIMBAD

\section{A. TABLES OF EMISSION LINE WAVELENGTHS, VELOCITIES, AND FLUXES}

Tables A1-A13 (available in the online journal) list the emission-line parameters for the high-resolution stars observed by COS and STIS that are the basis for this study. The data for $\alpha$ Cen A are published by Pagano et al. (2004), and the sources for the solar data are given in Section 2.4. The second column in each table is the laboratory wavelength given by Morton (1991) when available or by Pagano et al. (2004). The third and fourth columns give the measured wavelengths (and errors) and the corresponding radial velocities (and errors). The next two columns give the measured fluxes and FWHM of the lines with their measurement errors. For most of the emission lines, a single Gaussian provides a good fit to the data. For the brightest emission lines, we found that two Gaussians (a narrow and a broad Gaussian) provide a better fit to the data. For these bright lines, we list the parameters for both the narrow and broad Gaussian components described by the subscripts $n$ and $b$. The asterix superscript after the ion indicates that we computed a two-Gaussian fit to the emission line. For the same lines, we also provide the parameters for a single-Gaussian fit. These parameters are useful for comparison with fainter lines that can only be fit by a single Gaussian. Those lines marked $b l$ are close blends of lines from the same ion for which we use the mean laboratory wavelength. Widely separated blends or blends involving more than one atom or ion are not included.

We do not include very weak lines for which the flux errors exceed about $30 \%$ as the radial velocities of these lines are unreliable. We include flux upper limits for the coronal Fe XII $\lambda 1242.00$ and $\lambda 1349.36$ and Fe XXI $\lambda 1354.080$ lines when no emission feature is present. We have assumed that the FWHM $=45 \mathrm{~km} \mathrm{~s}^{-1}$ for the nondetected Fe XII lines and $110 \mathrm{~km} \mathrm{~s}^{-1}$ for the nondetected Fe XXI lines. For two stars (HII314 and HD 209458) the COS observations with the G130M and G160M gratings were taken at different times with the star likely placed in different portions of the aperture. For this reason the velocity scales for the two gratings are different. A solid horizontal line separates the data obtained with the different gratings. Table A5 lists the data for $\chi^{1}$ Ori obtained with COS and Table A6 lists the data obtained with STIS. 


\section{REFERENCES}

Achour, H., Brekke, P., Kjeldseth-Moe, O., \& Maltby, P. 1995, ApJ, 453, 945

Athay, R. G. \& Holzer, T. E. 1982, ApJ, 255, 743

Ayres, T. R. 1999, ApJ, 525, 240

Ayres, T. R. 2010, ApJS, 187, 149

Ayres, T., \& France, K. 2010, ApJ, 723, L38

Ayres, T. R., Brown, A., Harper, G. M., Osten, R. A., Linsky, J. L., Wood, B. E., \& Redfield, S. 2003, ApJ, 583, 963

Ayres, T. R., Marstad, N. C., \& Linsky, J. L. 1981, ApJ, 247, 545

Ayres, T. R., Simon, T., Stauffer, J. R., Stern, R. A., Pye, J. P., \& Brown, A. 1996, ApJ, 473,279

Barnes, S. A. 2007, ApJ, 669, 1167

Barnes, S. A. 2010, ApJ, 722, 222

Cardini, D., \& Cassatella, A. 2007, ApJ, 666, 393

Donati, J.-P. \& Landstreet, J. D. 2009, ARA\&A, 47, 333

Edlén, B. 1934, Nova Acta Reg. Soc. Sci. Ups. (IV), 9, No.6

France, K., Linsky, J. L., Brown, A., Froning, C. S., \& Béland, S. 2010a, ApJ, 715, 596

France, K., Stocke, J. T., Yang, H., Linsky, J. L., Wolven, B. C., Froning, C. S., Green, J. C., \& Osterman, S. N. 2010b, ApJ, 712, 1277

Gaidos, E. J., Henry, G. W., \& Henry, S. M. 2000, AJ, 120, 1006

Glebocki, R. \& Gnacinski, P. 2005, Catalog of Stellar Rotational Velocities, SIMBAD

Green, J. C. et al. 2012, ApJ, 744, 60

Hallin, R. 1966, Ark. Fys., 31, 511

Hansteen, V. H., Hara, H., De Pontieu, B., \& Carlsson, M. 2010, ApJ, 718, 1070 
Hempelmann, A., Schmitt, J. H. M. M., Schultz, M., Rüdiger, G., \& Stȩpień 1995, A\&A, 294,515

King, J. R., \& Schuler, S. C. 2005, PASP, 117, 911

Kraft, R. P. 1967, ApJ, 150, 551

Linsky, J. L., et al. 2010, ApJ, 717, 1291

Linsky, J. L., Ayres, T., Bushinsky, R., France, K., \& Fontenla, J. 2012, ApJ, 745, 25

Matt, S. \& Pudritz, R.E. 2008, ApJ, 681, 391

McClintock, W. E., Snow, M., \& Woods, T. N. 2005, Sol. Phys., 230, 259

Morton, D. C. 1991, ApJS, 77, 119

Noyes, R. W., Hartmann, L. W., Baliunas, S. L., Duncan, D. K., \& Vaughan, A. H. 1984, ApJ, 279, 763

Osterman, S. N., et al. 2011, Ap\&SS, 335, 2570

Pagano, I., Linsky, J. L., Valenti, J., \& Duncan, D. K. 2004, A\&A, 415, 331

Peter, H. 2001, A\&A, 374, 1108

Peter, H. 2006, A\&A, 449, 759

Peter, H. \& Judge, P. G. 1999, ApJ, 522, 1148

Peter, H., Gudiksen, B. V., \& Nordlund, A. 2006, ApJ, 638, 1086

Petit, P., Donati, J.-F., Aurière, M., Landstreet, J. D., Lignières, F., Marsden, S., Mouillet, D., Paletou, F., Toqué, N., \& Wade, G. A. 2005, MNRAS, 361, 837

Petit, P., et al. 2008, MNRAS, 388, 80

Pizzolato, N., Maggio, A., Micela, G., Sciortino, S., \& Ventura, P. 2003, A\&A, 397, 147

Radick, R. R., Thompson, D. T., Lockwood, G. W., Duncan, D. K., \& Baggett, W. E. 1987, ApJ, 321, 459

Redfield, S., Linsky, J. L., Ayres, T. R., \& Brown, A. 2001, in ASP Conf. Ser. 223, The $11^{\text {th }}$ Cool Stars, Stellar Systems, and the Sun, ed. R. J. Garcia Lopez, R. Rebolo, \& M. R. Zapatero Osorio (San Francisco:ASP), 1045 
Ribas, I., Guinan, E. F., Güdel, M., \& Audard, M. 2005, ApJ, 622, 680

Ribas, I., Porto de Mello, G. F., Ferreira, L. D., Hébrard, E., Selsis, F., Catalán, S., Garcès, A., do Nascimento, J. D., \& de Medeiros, J. R. 2010, ApJ, 714, 384

Rice, J. B., \& Strassmeier, K. G. 2001, A\&A, 377, 264

Schrijver, C. J. \& Title, A. M. 2001, ApJ, 551, 1099

Saar, S. H., \& Osten, R. A. 1997, MNRAS, 284, 803

Schroeder, C., Reiners, A., \& Schmitt, J. H. M. M. 2009, A\&A, 493, 1099

Silva-Valio, A. 2008, ApJ, 683, L179

Simon, T., Herbig, G., \& Boesgaard, A. M. 1985, ApJ, 293, 551

Skumanich, A. 1972, ApJ, 171, 565

Snow, M., McClintock, W. E., Rottman, G., \& Woods, T. N. 2005, Sol. Phys., 230, 295

Strassmeier, K. G., \& Rice, J. B. 1998, A\&A, 330, 685

Toner, C. G., \& Gray, D. F. 1988, ApJ, 334, 1008

Wilson, O. C. 1963, ApJ, 138, 832

This preprint was prepared with the AAS LATEX macros v5.2.

Wood, B. E., Linsky, J. L., \& Ayres, T. R. 1997, ApJ, 478, 745

Wood, B. E., Müller, H.-R., Zank, G. P., Linsky, J. L., \& Redfield, S. 2005, ApJ, 628, L143

Woods, T. N., et al. 2009, J. Geophys. Res., 36, L01101

Zacharias, P., Peter, H., \& Bingert, S. 2011, A\&A, 531, A97 


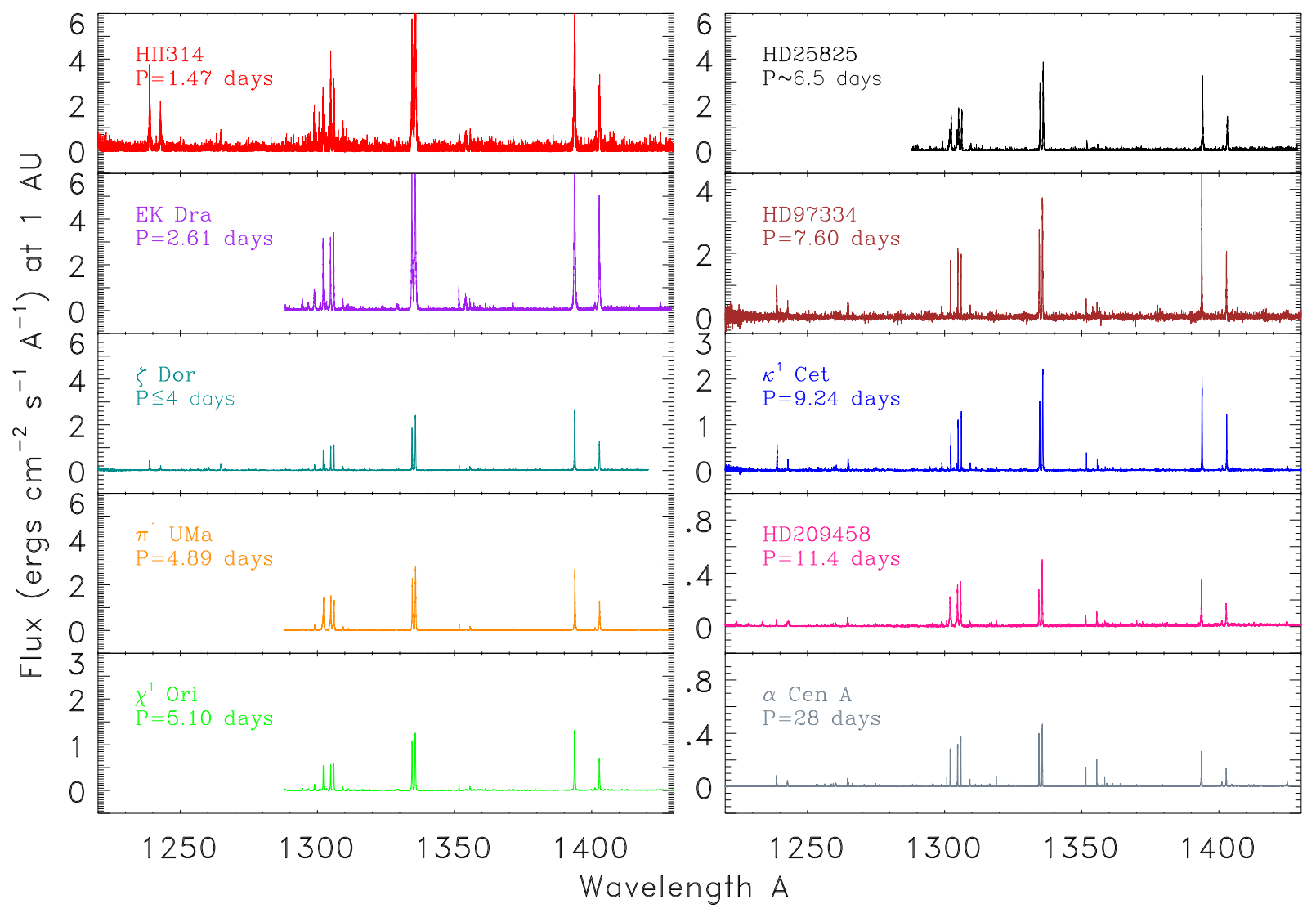

Fig. 1.- Comparison spectra of ten of the stars discussed in this paper. The stars are listed in order of decreasing rotation period $(\mathrm{P})$. The stars observed as a part of the SNAP program have a more limited spectral range. Note that the spectra of the slower rotating and thus older stars have different flux scales than the faster rotating stars. (A color version of this figure is available in the online journal.) 

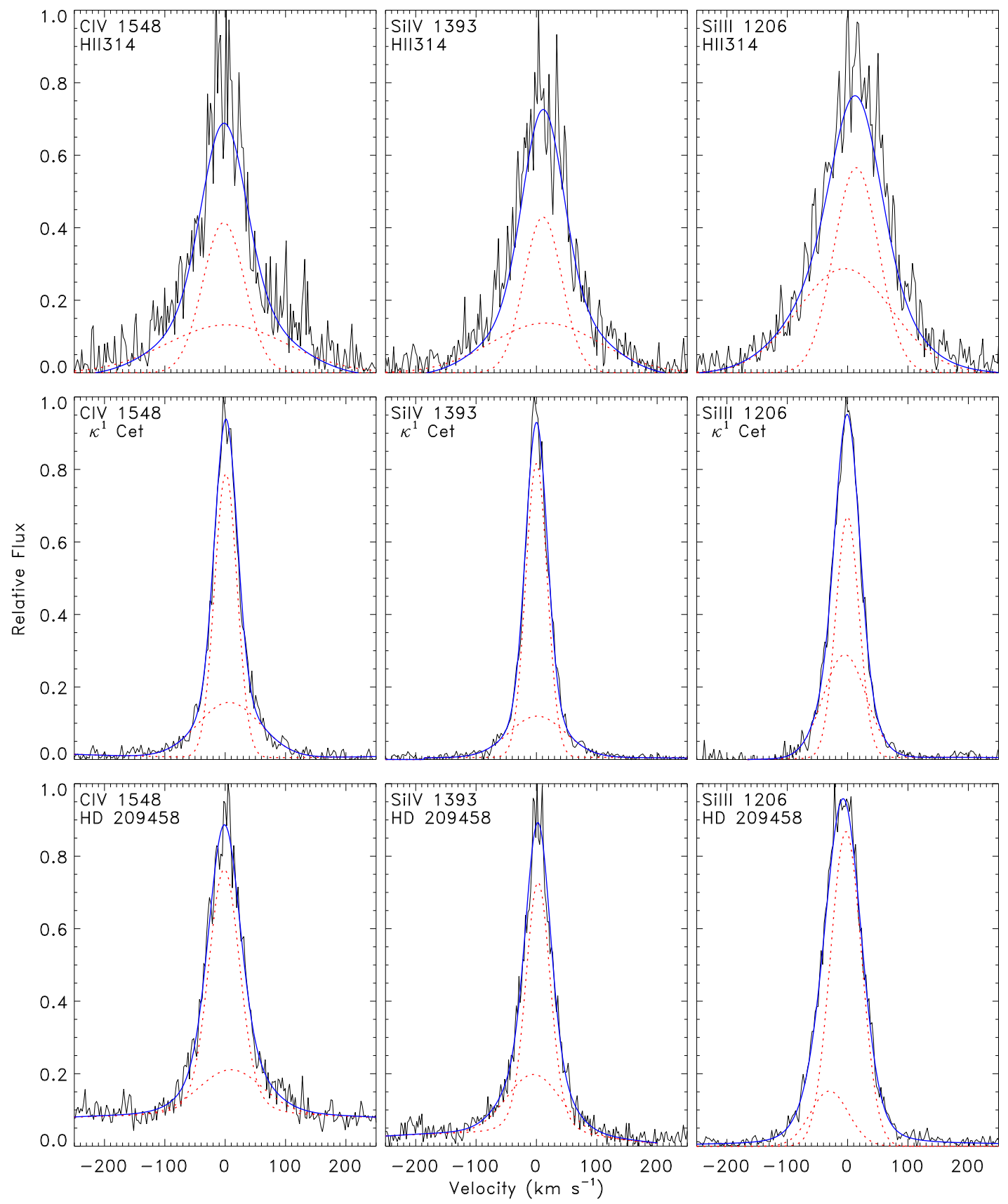

Fig. 2.- Two component fits to the C IV $\lambda 1548$, Si IV $\lambda 1393$, and Si III $\lambda 1206$ lines of three stars HII314, $\kappa^{1}$ Cet, and HD 209458. The narrow and broad component fits are shown as dotted (red) lines, and the solid (blue) lines are convolutions of the two Gaussians with the COS line-spread function that best fits the observed line profile. The broad components contain a large portion of the line fluxes for the rapid rotating star HII314 ( $P_{\text {rot }}=1.47$ days) but much smaller portions of the line fluxes for the more slowly rotating stars $\kappa^{1}$ Cet $\left(P_{\text {rot }}=8.77\right.$ days $)$ and HD 209458 ( $P_{\text {rot }}=11.4$ days $)$. (A color version of this figure is available in the online journal.) 


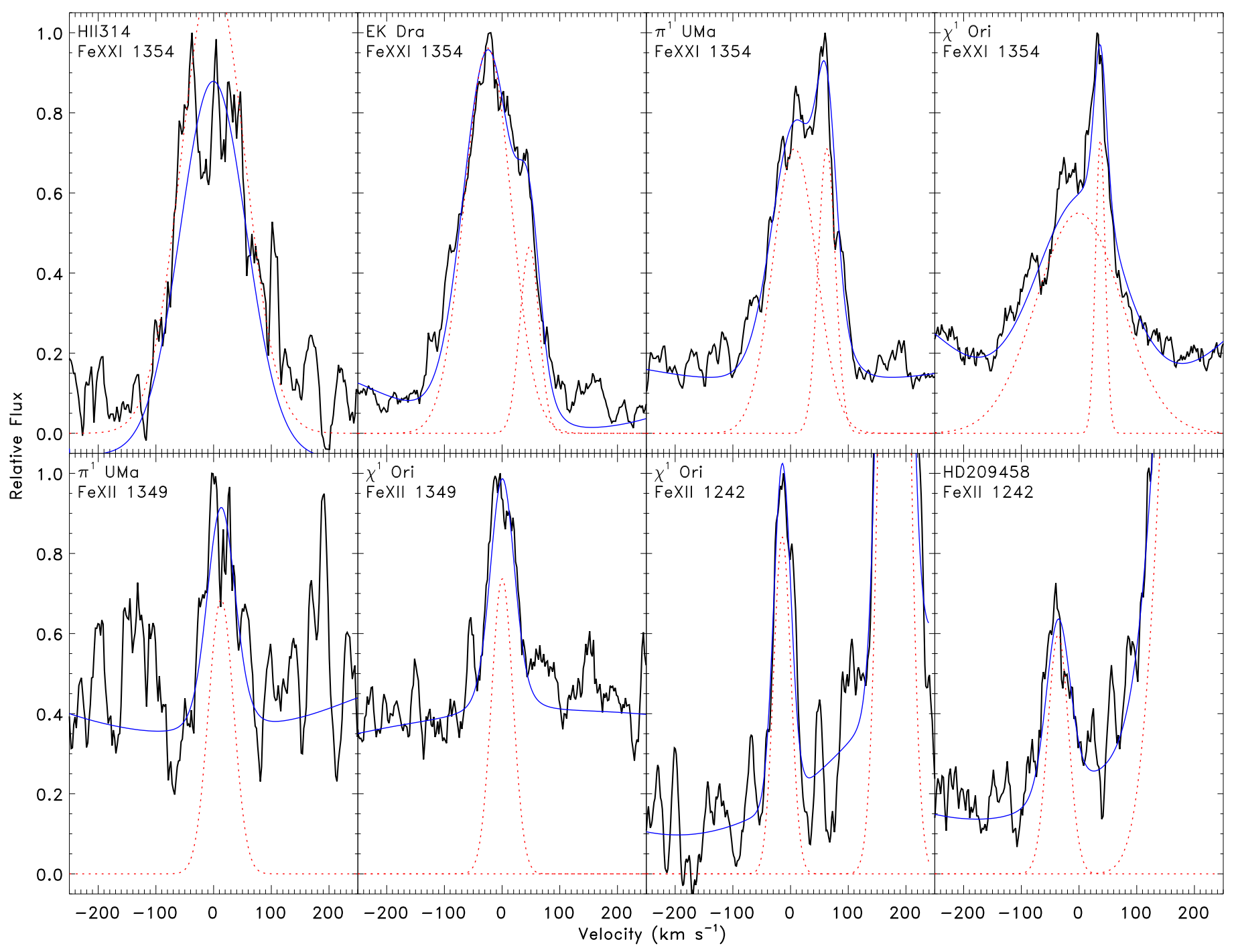

Fig. 3.- Comparison of the Fe XXI $\lambda 1354.080$ and Fe XII $\lambda 1242.000$ and $\lambda 1349.36$ lines observed in five stars. The C I $\lambda 1354.288$ line is identified and fit for three of the stars showing the Fe XXI line. With increasing rotation rate, the coronal Fe XXI line becomes brighter relative to the photospheric C I line. (A color version of this figure is available in the online journal.) 


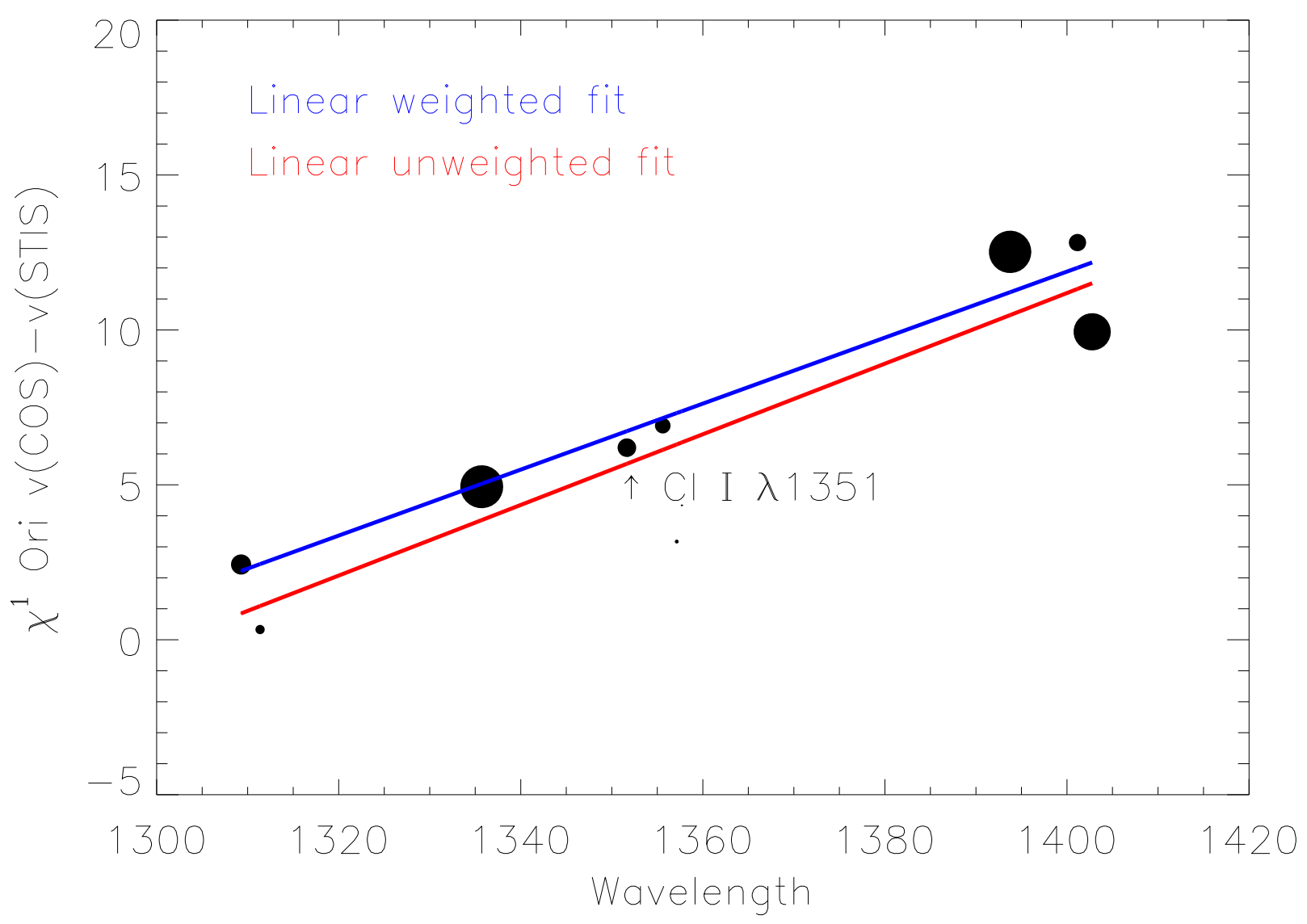

Fig. 4.- Radial velocity differences between the $\chi^{1}$ Ori COS and STIS measurements of the 9 emission lines in common with COS velocity-measurement errors less than $2.0 \mathrm{~km} \mathrm{~s}^{-1}$. The symbol sizes are proportional to the log of the COS line fluxes. Also plotted are fluxweighted and unweighted linear least-squares fits to the data. (A color version of this figure is available in the online journal.) 


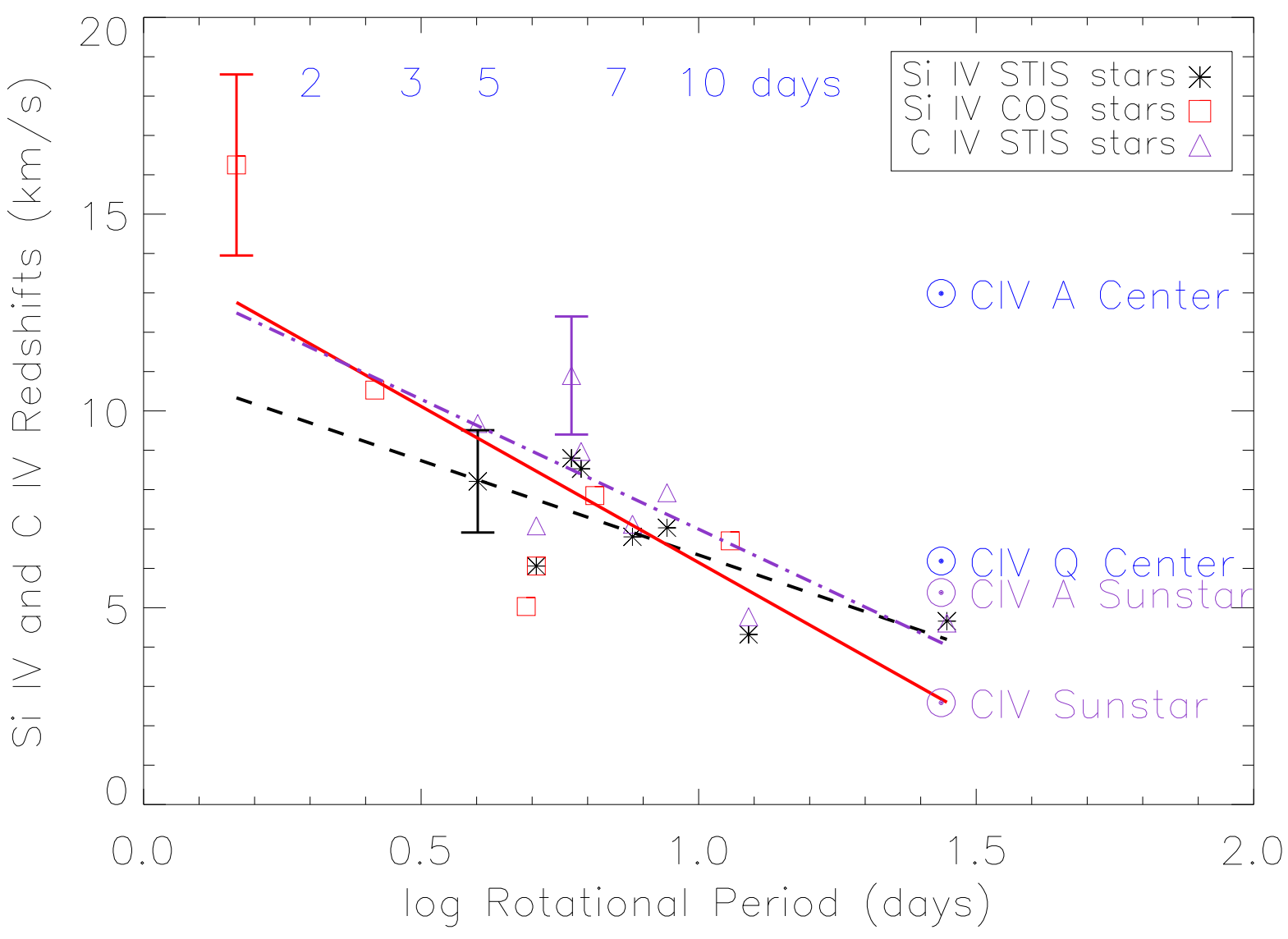

Fig. 5.- Redshifts of the Si IV and C IV lines plotted vs. stellar rotational period for solar-mass stars observed with the COS and STIS instruments on HST. The plotted Si IV velocities are flux-weighted averages of the central velocities of single-Gaussian fits to the $\lambda 1393$ and 1402 lines, except for the $\alpha$ Cen A velocities as described in Section 3. The Si IV velocities are measured relative to the photospheric Cl I $\lambda 1351$ line, and the COS Si IV velocities are shifted by $-5.45 \mathrm{~km} \mathrm{~s}^{-1}$ to be consistent with the STIS velocity scale for $\chi^{1}$ Ori (see Section 3). The C IV lines, all measured from STIS spectra, are flux-weighted averages of the $\lambda 1548$ and 1550 lines minus the $\mathrm{Cl} \mathrm{I}$ line velocity. The error bars are the mean errors for each data set: $2.3 \mathrm{~km} \mathrm{~s}^{-1}$ for the COS Si IV redshifts (larger for HII314), $1.5 \mathrm{~km} \mathrm{~s}^{-1}$ for the mean STIS Si IV redshifts, and $1.3 \mathrm{~km} \mathrm{~s}^{-1}$ for the STIS C IV redshifts. The dashed (black) line is the linear least squares fit to the STIS Si IV redshifts, the dash-dot (violet) line is the corresponding fit to the STIS C IV redshifts, and the solid (red) line is the fit to all of the Si IV redshifts. We include C IV redshifts for the solar disk-center quiet (CIV Q Center) and active (CIV A Center) regions, for the integrated disk (CIV Sunstar), and for the Sun covered with active regions (CIV A Sunstar, see Section 4). (A color version of this figure is available in the online journal.) 


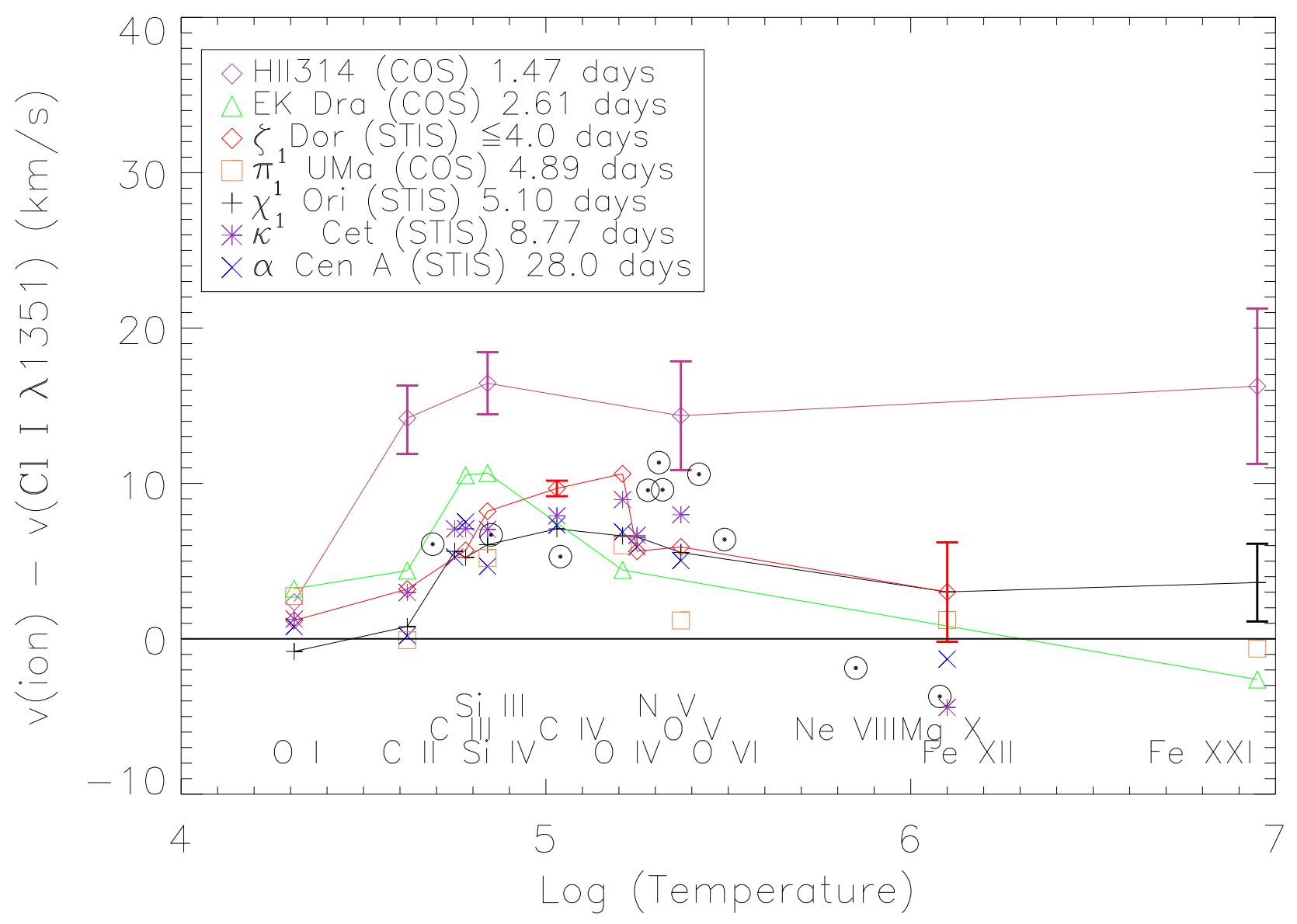

Fig. 6.- Emission-line redshifts relative to the $\mathrm{Cl}$ I $\lambda 1351$ line representing the stellar photosphere radial velocity. The sources for the data are listed in the legend, except that the Fe XXI velocity for $\chi^{1}$ Ori was obtained with COS. Typical measurement errors relative to the $\mathrm{Cl}$ I line are $0.3-0.8 \mathrm{~km} \mathrm{~s}^{-1}$ for the bright lines (e.g., O I, C II, Si III, Si IV, and C IV) and 1.5-3.0 $\mathrm{km} \mathrm{s}^{-1}$ for the faint lines (e.g., O IV and O V) for the stars observed with STIS and EK Dra and $\pi^{1}$ UMa observed with COS. The faintest star observed with COS, HII314, shows larger measurement errors of $1.3-1.7 \mathrm{~km} \mathrm{~s}^{-1}$ but $3.2 \mathrm{~km} \mathrm{~s}^{-1}$ for the faint O V $\lambda 1371$ line. Typical measurement errors for the Fe XII $\lambda 1242$ line observed by STIS are 2.5-3.7 km s${ }^{-1}$ and for the Fe XXI $\lambda 1354$ line are $2.5-5.0 \mathrm{~km} \mathrm{~s}^{-1}$ as shown in the figure. EK Dra ( $P_{\text {rot }}=2.61$ days) shows a similar redshift pattern to the slower rotators, but HII314 $\left(P_{\text {rot }}=1.47\right.$ days) shows an enhanced redshift pattern at all temperatures above $\log T=4.6$. Redshifts of the coronal lines (Fe XII and Fe XXI) are consistent with $0 \mathrm{~km} \mathrm{~s}^{-1}$ within the measurement errors except for HII314. The Sun symbols show solar disk-center redshift measurements of Peter \& Judge (1999) using the SUMER instrument on the SOHO spacecraft. (A color version of this figure is available in the online journal.) 


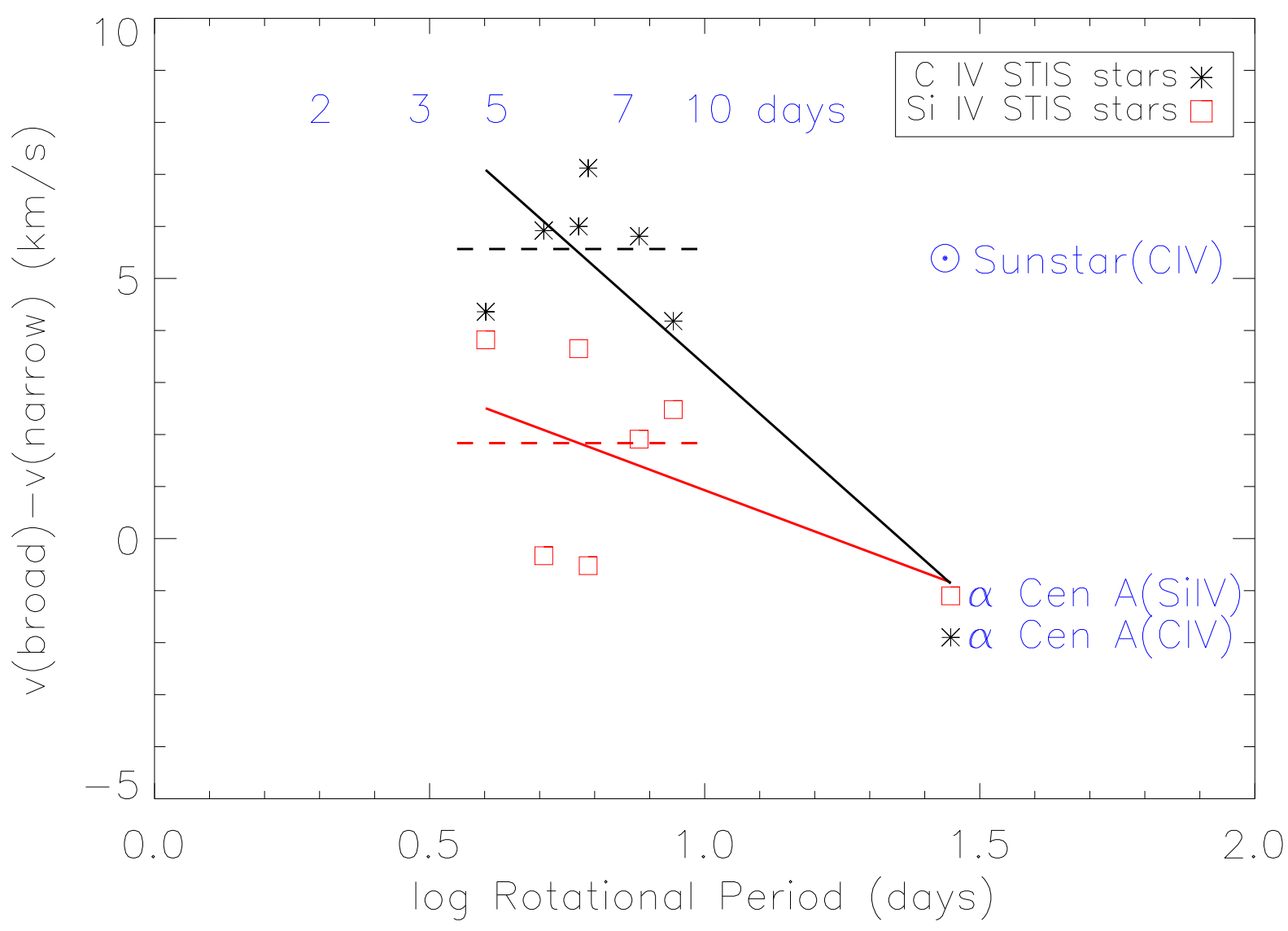

Fig. 7.- Velocity differences between the broad and narrow component centroids of the two Gaussian fits to the C IV $\lambda 1548$ and Si IV $\lambda 1393$ lines for the stars observed with STIS. Also shown is the velocity difference for the C IV line of the Sun viewed as a star (Sunstar). The mean values of the velocity differences (dashed lines) for all stars except $\alpha$ Cen A are $5.57 \mathrm{~km} \mathrm{~s}^{-1}$ for C IV and $1.82 \mathrm{~km} \mathrm{~s}^{-1}$ for Si IV. The Sunstar C IV velocity difference of 5.1 $\mathrm{km} \mathrm{s}^{-1}$ (Peter 2006) closely agrees with the stellar mean value. The solid lines are linear least-squares fits to all of the C IV and S IV stellar data, including $\alpha$ Cen A, which we believe are more realistic results but they should be tested by new data. (A color version of this figure is available in the online journal.) 
Table 1. Properties of the Solar Mass Stars Obtained with High Spectral Resolution

\begin{tabular}{|c|c|c|c|c|c|c|c|}
\hline Property/Star & HII314 & EK Dra & $\zeta$ Dor & $\pi^{1} \mathrm{UMa}$ & $\chi^{1}$ Ori & HD 165185 & HD 25825 \\
\hline Age $/ 10^{9}(\mathrm{yr})$ & 0.1 & $0.03-0.05$ & & 0.3 & 0.3 & 0.3 & 0.6 \\
\hline Cluster & Pleiades & Pleiades MG & & UMa MG & UMa MG & UMa MG & Hyades \\
\hline$P_{\text {rot }}($ days $)$ & 1.47 & 2.6050 & $<4.0$ & 4.89 & 5.10 & 5.90 & $\approx 6.5$ \\
\hline $\operatorname{vsini}(\mathrm{km} / \mathrm{s})$ & $38.7 \pm 1$ & $17.3 \pm 0.4$ & 14.8 & $9.7 \pm 0.4$ & $9.0 \pm 0.2$ & $7.6 \pm 1.2$ & 6.8 \\
\hline Sp Type & G1 V & G1.5 V & F9V & G1.5 V & G1 V & G1V & G0 V \\
\hline$R / R_{\odot}$ & 1.0 & 0.95 & 0.96 & 0.95 & 0.98 & 0.94 & 1.0 \\
\hline Other name & V1038 Tau & HD 129333 & HD 33262 & HD 72905 & HD 39587 & HIP 88694 & VB 10 \\
\hline $\mathrm{d}(\mathrm{pc})$ & 134 & 34 & 11.7 & 14.3 & 8.7 & 17.4 & 46.34 \\
\hline $\log L_{x}$ & 30.3 & 29.9 & & 29.1 & 29.0 & 29.17 & 28.9 \\
\hline$F_{x} / 10^{6}$ & 16 & 10 & & 2.0 & 2.0 & & 1.2 \\
\hline HST Spectrograph & COS G130M & COS G130M & STIS E140M & COS G130M & COS G130M & STIS E140M & COS G130M \\
\hline HST Program & 11532 & 11687 & 8280 & 11687 & 11687 & 9113 & 11687 \\
\hline Observation Date & $12 / 16 / 2009$ & $4 / 22 / 2010$ & $5 / 01 / 1999$ & $2 / 28 / 2010$ & $3 / 19 / 2010$ & $9 / 18 / 2002$ & $2 / 20 / 2010$ \\
\hline Exposure Time(sec) & 3209 & 1160 & 6000 & 1300 & 1300 & 3841 & 1160 \\
\hline Other observations & FOS,GHRS,IUE & GHRS,IUE,FUSE & STIS E230H & STIS E230H & STIS E140M,E230H & IUE & FOS \\
\hline
\end{tabular}


Table 2. Properties of the Solar Mass Stars Obtained with High Spectral Resolution

\begin{tabular}{|c|c|c|c|c|c|c|c|}
\hline Property/Star ${ }^{\mathrm{a}}$ & HD 97334 & $\kappa^{1}$ Cet & HD 73350 & HD 59967 & HD209458 & Sun & $\alpha$ Cen A \\
\hline $\begin{array}{l}\text { Age } / 10^{9}(\mathrm{yr}) \\
\text { Cluster }\end{array}$ & $0.45 \pm 0.20$ & 0.75 & $0.51 \pm 0.14$ & $0.35 \pm 0.07$ & $4 \pm 2$ & 4.6 & $3.9-4.9$ \\
\hline$P_{\text {rot }}$ (days) & 7.60 & 9.24 & $12.3 \pm 0.1$ & 6.14 & 11.4 & 25.4 & 28.0 \\
\hline $\operatorname{vsini}(\mathrm{km} / \mathrm{s})$ & 5.4 & 4.5 & $4.0 \pm 0.5$ & 3.8 & 4.5 & 1.7 & 3.2 \\
\hline Sp Type & G0 V & G5 V & G0 & G3 V & G0 V & G2 V & $\mathrm{G} 2 \mathrm{~V}$ \\
\hline$R / R_{\odot}$ & 1.01 & 0.99 & 1.07 & 0.89 & 1.125 & 1.00 & 1.224 \\
\hline Other name & MN UMa & HD 20630 & HIP 42333 & HIP 36515 & V376 Peg & - & HD 128620 \\
\hline $\mathrm{d}(\mathrm{pc})$ & 23.8 & 9.2 & 23.6 & 21.8 & 47 & - & 1.338 \\
\hline $\log L_{x}$ & 29.1 & 28.8 & & & & $26.3-27.3$ & $26.5-27.0$ \\
\hline$F_{x} / 10^{6}$ & 1.8 & 1.0 & & & & $0.0002-0.002$ & $0.003-0.01$ \\
\hline HST Spectrograph & STIS E140M & STIS E140M & STIS E140M & STIS E140M & COS G130M & SORCE & STIS E140H \\
\hline HST Program & 9113 & 8280 & 9113 & 9113 & 11534 & - & 7263 \\
\hline Observation Date & $6 / 21 / 2003$ & $9 / 19 / 2000$ & $4 / 26 / 2003$ & $9 / 10 / 2003$ & $9+10 / 2009$ & $3 / 2008$ & 2/12/1999 \\
\hline Exposure Time(sec) & 3841 & 7805 & 3841 & 3841 & 20694 & 180 & 14085 \\
\hline Other observations & IUE & STIS E230H & - & - & STIS,GHRS & SOHO/SUMER & GHRS,FUSE \\
\hline
\end{tabular}

a Age references: Plavchan et al. (2009), Ribas et al. (2010)

$P_{\text {rot }}$ and vsini references: Pizzolato et al. (2003), Gaidos et al. (2000), Petit et al. (2008). 
Table 3. Observing Log of Eight Stars

\begin{tabular}{lccccccr}
\hline \hline Star $^{\mathrm{a}}$ & Instrument & Dataset & Grating & Central & Yr-mn-day & Start Time & $\mathrm{T}_{\text {exp }}(\mathrm{s})$ \\
\hline HII314 & COS & LB6401010 & G130M & 1309 & $2009-12-12$ & $16: 09: 28$ & 814.1 \\
HII314 & COS & LB6401020 & G130M & 1318 & $2009-12-12$ & $16: 09: 45$ & 990.1 \\
HII314 & COS & LB6401040 & G130M & 1300 & $2009-12-12$ & $16: 11: 14$ & 1405.2 \\
HII314 & COS & LB6402010 & G160M & 1600 & $2010-10-11$ & $11: 49: 04$ & 1402.0 \\
HII314 & COS & LB6402020 & G160M & 1577 & $2010-10-11$ & $12: 15: 32$ & 326.9 \\
EK Dra & COS & LB3E34010 & G130M & 1291 & $2010-04-22$ & $09: 18: 42$ & 1160.0 \\
$\pi^{1}$ UMa & COS & LB3E26010 & G130M & 1291 & $2010-02-28$ & $22: 31: 16$ & 1300.4 \\
$\chi^{1}$ Ori & COS & LB3E06010 & G130M & 1291 & $2010-03-19$ & $06: 47: 26$ & 1300.4 \\
$\chi^{1}$ Ori & STIS & O5BN02010-30 & E140M & 1425 & $2000-10-03$ & $01: 11: 14$ & 6770.0 \\
HD25825 & COS & LB3E41010 & G130M & 1291 & $2010-02-20$ & $23: 15: 33$ & 1160.0 \\
$\kappa^{1}$ Cet & STIS & O5BN03050-60 & E140M & 1425 & $2000-09-19$ & $08: 58: 48$ & 7805.0 \\
HD165185 & STIS & O6CO07040 & E140M & 1425 & $2001-09-18$ & $00: 05: 52$ & 2977.0 \\
HD165185 & STIS & O6CO07050 & E140M & 1425 & $2001-09-17$ & $23: 11: 23$ & 864.0 \\
HD73350 & STIS & O6CO06040 & E140M & 1425 & $2002-04-26$ & $03: 32: 25$ & 2899.0 \\
HD73350 & STIS & O6CO06050 & E140M & 1425 & $2002-04-26$ & $02: 30: 59$ & 864.0 \\
\hline
\end{tabular}

${ }^{\text {a}}$ For information on the observations of $\alpha$ Cen A see Pagano et al. (2004). For information on the observations of HD 209458 see Linsky et al. (2010) and France et al. (2010a). 
Table 4. Radial Velocities for $\chi^{1}$ Ori (STIS data)

\begin{tabular}{lccc}
\hline \hline $\begin{array}{l}\text { Atom } \\
\text { or Ion }\end{array}$ & $\begin{array}{c}\text { Number } \\
\text { of Lines }\end{array}$ & $\begin{array}{c}<v \pm 1 \sigma^{\mathrm{a}} \\
\left(\mathrm{km} \mathrm{s}^{-1}\right)\end{array}$ & $\begin{array}{c}\text { Total flux } \\
\left(10^{-15}\right)\end{array}$ \\
\hline Cl I & 1 & $-15.51 \pm 0.35$ & 10.49 \\
S I & 9 & $-15.17 \pm 0.79$ & 23.18 \\
Si I & 9 & $-16.56 \pm 0.76$ & 35.07 \\
C I & 21 & $-15.96 \pm 0.42$ & 60.50 \\
N I & 2 & $-15.04 \pm 0.76$ & 5.25 \\
O I & 3 & $-16.32 \pm 0.06$ & 156.07 \\
N II & 3 & $-15.14 \pm 3.69$ & 4.85 \\
Fe II & 33 & $-15.37 \pm 0.47$ & 218.97 \\
Si II & 17 & $-15.50 \pm 0.55$ & 169.94 \\
\hline & & & \\
Sum S I to Si II & 97 & $-15.77 \pm 0.17$ & 673.83 \\
& & & \\
\hline C II & 2 & $-14.91 \pm 0.23$ & 359.16 \\
He II & 1 & $-11.10 \pm 0.23$ & 258.45 \\
S III & 1 & $-11.48 \pm 1.79$ & 46.70 \\
Si III & 7 & $-13.69 \pm 0.53$ & 378.67 \\
C III & 3 & $-9.87 \pm 1.21$ & 72.66 \\
N IV & 1 & $-12.66 \pm 2.57$ & 33.80 \\
O IV] & 3 & $-10.00 \pm 0.17$ & 106.76 \\
Si IV & 2 & $-9.45 \pm 0.34$ & 302.14 \\
C IV & 2 & $-7.53 \pm 1.95$ & 390.63 \\
N V & 2 & $-11.71 \pm 0.20$ & 46.16 \\
O V & 2 & $-9.95 \pm 0.29$ & 30.25 \\
Fe XII & & & \\
\hline
\end{tabular}

${ }^{\mathrm{a}} \sigma$ is the standard error of the weighted-mean radial velocity when more than one line is available or the error in the centriod of the line profile when there is only one line. 
Table 5. Emission Line Redshifts Using the Cl I $1351 \AA$ Line as a Fiducial for the Photospheric Radial Velocity

\begin{tabular}{|c|c|c|c|c|c|c|c|c|c|}
\hline Star & $\begin{array}{l}\text { Spectral } \\
\text { Type }\end{array}$ & Instrument $^{\mathrm{a}}$ & $\begin{array}{c}P_{\text {rot }} \\
\text { (days) }\end{array}$ & $\mathrm{v}(\mathrm{Si} I V)$ & $\mathrm{v}(\mathrm{C}$ IV $)$ & $\mathrm{v}(\mathrm{Cl} \mathrm{I})$ & $\begin{array}{c}\mathrm{v}(\mathrm{Si} \text { IV }) \\
\mathrm{COS}\end{array}$ & $\begin{array}{c}-\mathrm{v}(\mathrm{Cl} \mathrm{I}) \\
\mathrm{STIS}\end{array}$ & $\begin{array}{c}\mathrm{v}(\mathrm{C} \text { IV })-\mathrm{v}(\mathrm{Cl} \mathrm{I}) \\
\text { STIS }\end{array}$ \\
\hline HII314 & G1 V & COS & 1.47 & -1.69 & & -23.95 & +21.70 & +16.25 & \\
\hline EK Dra & G1.5 V & COS & 2.61 & -1.46 & & -17.44 & +15.98 & +10.53 & \\
\hline$\zeta$ Dor & F9 V & STIS & $\leq 4.0$ & +6.54 & +8.01 & -1.67 & & +8.21 & +9.68 \\
\hline$\pi^{1} \mathrm{UMa}$ & G1.5 V & COS & 4.89 & +22.91 & & +12.43 & +10.48 & +5.03 & \\
\hline$\chi^{1}$ Ori & G1 V & COS & 5.10 & +2.20 & & -9.31 & +11.51 & +6.06 & \\
\hline$\chi^{1}$ Ori & G1 V & STIS & 5.10 & -9.45 & -8.43 & -15.51 & & +6.06 & +7.08 \\
\hline HD 165185 & G1 V & STIS & 5.90 & +23.18 & +25.28 & +14.38 & & +8.80 & +10.90 \\
\hline HD 59967 & G3 V & STIS & 6.14 & +14.05 & +14.49 & +5.52 & & +8.53 & +8.97 \\
\hline HD 25825 & G0 V & $\mathrm{COS}$ & $\approx 6.5$ & +65.72 & & +52.42 & +13.30 & +7.85 & \\
\hline HD 97334 & G0 V & STIS & 7.60 & +2.32 & +2.64 & -4.48 & & +6.80 & +7.12 \\
\hline$\kappa^{1}$ Cet & G5 V & STIS & 8.77 & +24.11 & +25.00 & +17.08 & & +7.03 & +7.92 \\
\hline HD 209458 & G0 V & COS & 11.4 & -18.75 & & -30.90 & +12.15 & +6.70 & \\
\hline HD 73350 & G0 & STIS & 12.3 & +38.96 & +39.41 & +34.64 & & +4.32 & +4.77 \\
\hline Quiet Sun & $\mathrm{G} 2 \mathrm{~V}$ & SUMER & 25.4 & & & & & $\approx 2.3$ & $\approx 2.6$ \\
\hline Disk Center & & HRTS & & & & & & +5.4 & +6.2 \\
\hline Active Sun & $\mathrm{G} 2 \mathrm{~V}$ & SUMER & 25.4 & & & & & $\approx 4.6$ & $\approx 5.4$ \\
\hline Disk Center & & HRTS & & & & & & +11.0 & +13.0 \\
\hline$\alpha$ Cen A & $\mathrm{G} 2 \mathrm{~V}$ & STIS & 28.0 & -17.51 & -17.56 & -22.17 & & +4.7 & +4.6 \\
\hline
\end{tabular}


${ }^{a}$ STIS velocities were measured from the StarCAT database. COS velocity differences $(\mathrm{v}(\mathrm{Si} \mathrm{IV})-\mathrm{v}(\mathrm{Cl} \mathrm{I})$ ) are corrected to the STIS scale (in STARCat) by subtracting $5.45 \mathrm{~km} \mathrm{~s}^{-1}$ based on the comparison of $\chi^{1}$ Ori COS and STIS spectra. We use the centroid velocities of single Gaussian fits to the emission lines. 
Table A1. Emission Line Profile Parameters ${ }^{\mathrm{a}}$ for HII314 (COS)

\begin{tabular}{|c|c|c|c|c|c|c|c|c|c|}
\hline $\operatorname{Ion}^{\mathrm{b}}$ & $\begin{array}{c}\lambda_{l a b} \\
(\AA)\end{array}$ & $\begin{array}{l}\lambda_{n} \\
(\AA)\end{array}$ & $\begin{array}{c}\Delta v_{n} \\
\left(\mathrm{~km} \mathrm{~s}^{-1}\right)\end{array}$ & $\begin{array}{c}\text { Flux }_{n} \\
\left(10^{-16}\right)\end{array}$ & $\begin{array}{l}\mathrm{FWHM}_{n} \\
\left(\mathrm{~km} \mathrm{~s}^{-1}\right)\end{array}$ & $\begin{array}{c}\lambda_{b} \\
(\AA)\end{array}$ & $\begin{array}{c}\Delta v_{b} \\
\left(\mathrm{~km} \mathrm{~s}^{-1}\right)\end{array}$ & $\begin{array}{c}\text { Flux }_{b} \\
\left(10^{-16}\right)\end{array}$ & $\begin{array}{l}\mathrm{FWHM}_{b} \\
\left(\mathrm{~km} \mathrm{~s}^{-1}\right)\end{array}$ \\
\hline Fe XXI & 1354.080 & $1354.0268 \pm 0.0073$ & $-6.86 \pm 1.61$ & $4.25 \pm 0.70$ & $138.6 \pm 10.8$ & & & & \\
\hline Fe XII & 1242.000 & & & $<0.32$ & $(45.0)$ & & & & \\
\hline Fe XII & 1349.36 & & & $<0.14$ & $(45.0)$ & & & & \\
\hline $\mathrm{O} \mathrm{V}$ & 1218.344 & $1218.3437 \pm 0.0122$ & $-0.07 \pm 3.01$ & $2.31 \pm 0.33$ & $83.7 \pm 9.4$ & & & & \\
\hline $\mathrm{OV}$ & 1371.292 & $1371.2608 \pm 0.0144$ & $-6.82 \pm 3.16$ & $1.06 \pm 0.33$ & $46.7 \pm 8.9$ & & & & \\
\hline $\mathrm{N} \mathrm{V}$ & 1238.821 & $1238.7850 \pm 0.0082$ & $-8.71 \pm 1.98$ & $17.13 \pm 0.99$ & $118.3 \pm 4.3$ & & & & \\
\hline $\mathrm{N} \mathrm{V}$ & 1242.804 & $1242.7803 \pm 0.0130$ & $-5.71 \pm 3.13$ & $5.62 \pm 0.68$ & $87.7 \pm 6.8$ & & & & \\
\hline $\mathrm{Si} \mathrm{IV}^{*}$ & 1393.755 & $1393.7440 \pm 0.0072$ & $-2.36 \pm 1.55$ & $27.07 \pm 1.94$ & $83.9 \pm 4.5$ & $1393.6162 \pm 0.0317$ & $-29.85 \pm 6.82$ & $14.92 \pm 2.87$ & $270.5 \pm 35.1$ \\
\hline Si IV & 1393.755 & $1393.7424 \pm 0.0065$ & $-2.71 \pm 1.40$ & $32.05 \pm 1.67$ & $94.2 \pm 3.4$ & & & & \\
\hline Si IV & 1402.770 & $1402.7735 \pm 0.0042$ & $+0.75 \pm 0.90$ & $13.91 \pm 0.52$ & $93.7 \pm 2.6$ & & & & \\
\hline Si III* & 1206.50 & $1206.4711 \pm 0.0080$ & $-7.18 \pm 2.00$ & $27.09 \pm 11.04$ & $97.2 \pm 14.2$ & $1206.45 .63 \pm 0.0184$ & $-10.86 \pm 4.58$ & $20.92 \pm 18.55$ & $171.6 \pm 53.2$ \\
\hline Si III & 1206.50 & $1206.4693 \pm 0.0057$ & $-7.63 \pm 1.42$ & $49.62 \pm 2.10$ & $130.7 \pm 3.9$ & & & & \\
\hline C II & 1335.7077 & $1335.6583 \pm 0.0060$ & $-11.09 \pm 1.35$ & $42.64 \pm 1.93$ & $103.8 \pm 3.2$ & & & & \\
\hline O I & 1304.8576 & $1304.7382 \pm 0.0073$ & $-27.43 \pm 1.67$ & $6.39 \pm 1.08$ & $42.7 \pm 4.8$ & & & & \\
\hline O I & 1306.0286 & $1305.9190 \pm 0.0076$ & $-25.16 \pm 1.75$ & $5.95 \pm 0.98$ & $42.0 \pm 4.3$ & & & & \\
\hline $\mathrm{Cl} \mathrm{I}$ & 1351.6561 & $1351.5507 \pm 0.0071$ & $-23.39 \pm 1.57$ & $1.93 \pm 0.23$ & $46.4 \pm 3.2$ & & & & \\
\hline $\mathrm{C} \mathrm{IV}^{*}$ & 1548.195 & $1548.3087 \pm 0.0104$ & $+22.01 \pm 2.02$ & $49.02 \pm 6.92$ & $61.9 \pm 6.9$ & $1548.3772 \pm 0.0217$ & $+35.27 \pm 4.19$ & $84.78 \pm 12.27$ & $222.1 \pm 22.2$ \\
\hline C IV & 1548.195 & $1548.3071 \pm 0.0089$ & $+21.71 \pm 1.72$ & $95.30 \pm 5.74$ & $108.9 \pm 4.7$ & & & & \\
\hline $\mathrm{C} \mathrm{IV}^{*}$ & 1550.770 & $1550.9379 \pm 0.0083$ & $+32.48 \pm 1.61$ & $32.90 \pm 2.90$ & $75.3 \pm 5.1$ & $1550.9140 \pm 0.0229$ & $+27.85 \pm 4.43$ & $33.43 \pm 4.18$ & $218.8 \pm 12.1$ \\
\hline CIV & 1550.770 & $1550.9272 \pm 0.0106$ & $+30.39 \pm 2.05$ & $53.96 \pm 3.69$ & $105.5 \pm 4.7$ & & & & \\
\hline He II & 1640.400 & $1640.5442 \pm 0.0069$ & $+26.37 \pm 1.26$ & $107.26 \pm 6.05$ & $80.8 \pm 2.7$ & & & & \\
\hline C I & 1656.2672 & $1656.3593 \pm 0.0099$ & $+16.68 \pm 1.79$ & $10.25 \pm 1.22$ & $52.2 \pm 3.6$ & & & & \\
\hline
\end{tabular}

${ }^{a}$ COS G130M data are above the break line and COS G160M data taken later are below the break line. Parameters for the narrow components have an "n" subscript and parameters for the broad components have a "b" subscript.

${ }^{\mathrm{b}}$ Spectral lines with the ${ }^{*}$ symbol are preferred one-Gaussian or two-Gaussian fits to the observed data. Single Gaussian fits are listed below the two-component fits. 